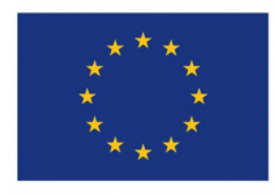

\title{
Recruiting Research Participants through Facebook Advertisements: A Handbook*
}

\author{
Anja Neundorf (University of Glasgow) \\ Aykut Öztürk (University of Glasgow)
}

Version: December 9, 2021

To cite: Neundorf, A., \& Öztürk, A. (2021, December 9). Recruiting Research Participants through Facebook Advertisements: A Handbook. Retrieved from https://doi.org/10.31219/osf.io/87rg3

\footnotetext{
*For feedback, suggestions or questions on this handbook, please contact us on demedproject@glasgow.ac.uk or anja.neundorf@glasgow.ac.uk. This handbook might be updated based on the changes in Facebook's tools and the feedback we will receive; please make sure that you have the most recent version by checking the DOI provided above. This handbook is based on a webinar series, which ran in Nov-Dec 2021. Additional material and video recordings can be accessed here: https: //www.gla.ac.uk/research/az/democracyresearch/datamethods/facebookasaresearchtool/. This research was generously funded by the ERC Consolidator Grant DEMED (grant number: 865305).
} 


\section{Contents}

1 Introduction 3

1.1 Understanding Facebook campaigns . . . . . . . . . . . . . . . . . . . . 4

2 Basic set-up: Facebook Ads Manager, Events Manager and your Face$\begin{array}{ll}\text { book Page } & 7\end{array}$

3 Setting-up a conversion infrastructure $\quad 11$

3.1 Creating a Facebook Pixel . . . . . . . . . . . . . . . . . . . . 13

3.2 Installing Facebook Pixel to your website . . . . . . . . . . . . . . 14

3.3 Defining an event . . . . . . . . . . . . . . . . 17

3.4 Verifing domain ownership of your website . . . . . . . . . . 18

3.5 Prioritizing your events . . . . . . . . . . . . . . . . . 19

4 Creating advertisement campaigns $\quad 21$

4.1 The design of Facebook Ads Manager . . . . . . . . . . . . . . . . . . . . 21

4.2 How to create an advertisement campaign . . . . . . . . . . . . . 23

4.2.1 Choices at campaign level . . . . . . . . . . . . . 24

4.2.2 Choices at ad set level . . . . . . . . . . . . . . 26

4.2 .3 Choices at ad level . . . . . . . . . . . . . . . . 29

4.3 Using targeting tools to create more representative samples . . . . . . . 31

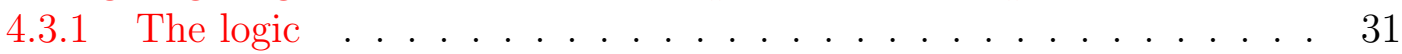

4.3.2 The practice. . . . . . . . . . . . . . . 33

4.4 Setting URL parameters to know where your survey respondents are coming from . . . . . . . . . . . . . . . . . . . 35

5 Bibliography $\quad 38$ 


\section{Introduction}

Using paid advertisements on Facebook has proven to be one of the most effective online advertisement methods, which can be a useful tool for social science researchers. Advertisers can purchase advertisement space from Meta Inc. to show their advertisements on Facebook, Instagram, or Facebook Messenger. ${ }^{1}$ An example Facebook paid advertisement, which we used in our own advertisement campaigns, can be seen in Figure 1. Most advertisement campaigns on Facebook are designed such that Facebook users clicking on the advertisement campaign is taken to the web page that advertiser wants. In our studies we use Facebook paid advertisements to direct social media users to our survey page, hosted on Qualtrics platform, to take our surveys.

Figure 1: An example advertisement

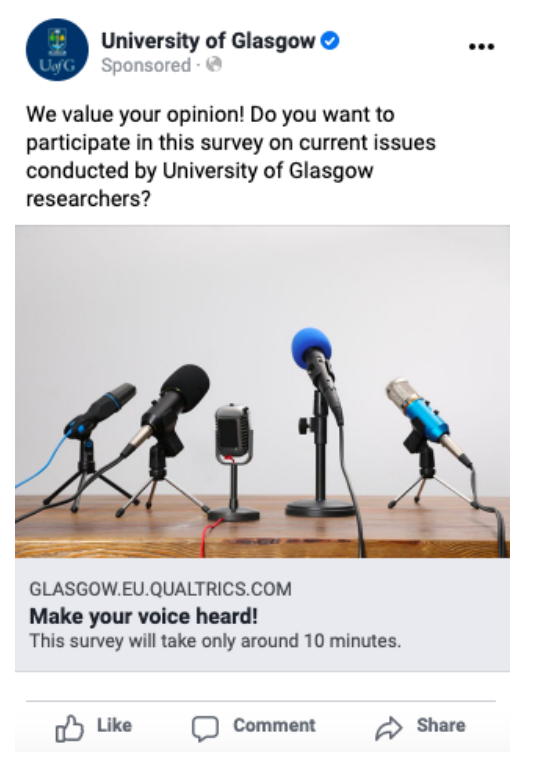

Using paid Facebook advertisements to recruit survey participants is appealing for several reasons. First, it provides direct and easy access to a very large pool of survey respondents. Facebook boasts a user base of 2.83 billion, making it the most widely used

\footnotetext{
${ }^{1}$ Throughout the document we mainly refer to Facebook. But all actions taken and described include all Meta Inc. platforms using paid advertisement. As we will explain in more detail to set-up and manage an advertisement campaign on the different platform only the Facebook Advertisement Manager is needed.
} 
social media company in the world. As seen in Figure 2, significant portions of population in both rich and poor, both democratic and non-democratic countries visit Facebook, at least once a month. ${ }^{2}$ The design of Facebook advertisement system allows researchers to immediately start recruiting survey participants from this large pool, without needing to deal with survey companies. Secondly, participants recruited through Facebook advertisements cost significantly less than participants recruited through other means. For example, our surveys in the UK costed as little as $£ 0.25(\$ 0.33)$ per complete response while we were charged $£ 4.45(\$ 5.90)$ per complete response by a commercial survey company for a similar survey in the same country. Finally, targeting tools available with Facebook allows researchers to target populations or specific groups that would be hard to reach, such as refugees or specific occupational groups).

Figure 2: Proportion of the population using Facebook at least once a month

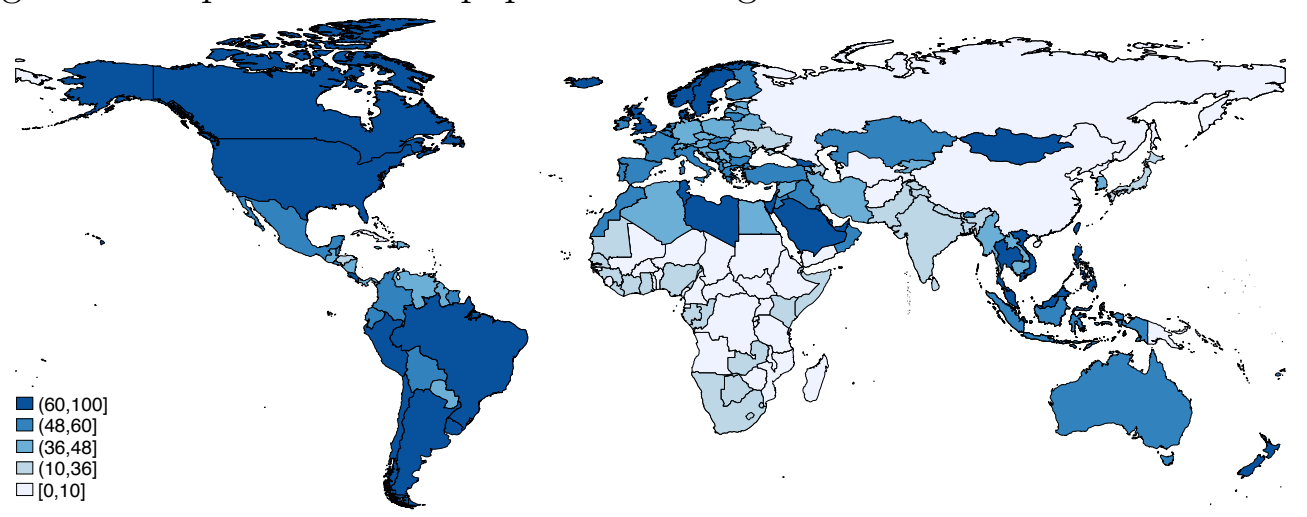

\subsection{Understanding Facebook campaigns}

Facebook advertisement can be divided into three main so called campaigns, which rely on different algorithms to distribute ads on the various platforms: Reach, traffic and conversion. All advertisement campaigns have the same basic set-up on Facebook: Advertisers will visit the Facebook Ads Manager, determine the audience and content of

\footnotetext{
${ }^{2}$ Source: https://worldpopulationreview.com/country-rankings/facebook-users-by-country, accessed April 8, 2021.
} 
the advertisements, and publish them. Using these ads for the recruitment of research participants, the aim is for social media users to click on the ad, which then directs the user to an external page, where a survey is hosted (e.g. Qualtrics, SurveyMonkey, Google Forms).

Conversion campaigns are advertisement campaigns in which the Facebook algorithms intend to maximize the number of user actions taken outside of the Facebook environment, such as adding an item to the shopping cart in the advertiser's web page or as is used in research, completing a survey. These campaigns track the behavior of Facebook users, who clicked on the advertisement link, outside of the Facebook environment using so-called pixels. Hence if you want to use conversion campaigns you will first need to set-up the infrastructure that will allow data transfer between your Facebook account and web pages that will be tracked for user behavior. This process can take up to one or two hours and will be explained in detail in this handbook. Once you set-up the necessary infrastructure, you can use it any time you create a new advertisement campaign.

Traffic campaigns aim to maximize the number of Facebook users who will click on an advertisement. In other words, these campaigns aim to increase the "traffic" to your web page. For traffic campaigns, Facebook only tracks behavior taken within the Facebook environment. Hence, advertisers do not need to spend time setting-up a data transfer infrastructure if they will only use traffic campaigns.

Finally, reach campaigns aim to maximize the number of Facebook users who will see the advertisement or the number of times Facebook users will see the advertisement. Similar to traffic campaigns, reach campaigns do not track behavior outside of Facebook environment. This means that advertisers setting up reach campaigns do not need to set-up a data transfer infrastructure. 
As we have demonstrated in our research, conversion campaigns are most effective for using Facebook as a research tool (Neundorf \& Öztürk, 2021). Conversion campaigns recruited new research participants for, at least, $30 \%$ smaller costs than traffic campaigns. The cost difference was up to ten times higher in some countries. Reach campaigns, on the other hand, were the least successful campaigns. Detailed analysis of our results have shown that, although reach campaigns can create more views of your advertisements and traffic campaigns can drive more traffic to your survey page, conversion campaigns are more successful at recruiting Facebook users who will complete your survey.

For this reason, we recommend the use of conversion campaigns for participant recruitment purposes. Traffic campaigns can also be useful if you are planning to recruit only a small number of participants and you believe that most Facebook users visiting your survey page will complete your survey. We do not see any conditions under which reach campaigns would be useful for recruitment purposes. After reading this handbook, you will be able to set-up all three kinds of campaigns.

This handbook is formed of five sections. Following this section, Section 2 introduces web pages that you will need to use to create advertisements on Facebook and what you need to do to be able to use those web pages. In Section 3, we describe steps that you need to complete to set-up your conversion infrastructure. If you will only use traffic campaigns, you can skip this section and go directly to Section 4. In Section 4, we demonstrate how to create advertisement campaigns on Facebook. These steps are, more or less, the same for all forms of advertisement campaigns on Facebook. This section also discusses how you can modify your advertisement campaign to recruit more representative samples and how you can set URL parameters to record which advertisement each of your survey participant is coming from. Finally, in Section 5, we present a list of academic articles that explore best practices while using Facebook ads 
to recruit research participants.

\section{Basic set-up: Facebook Ads Manager, Events Manager and your Facebook Page}

Figure 3: Facebook Ads Manager

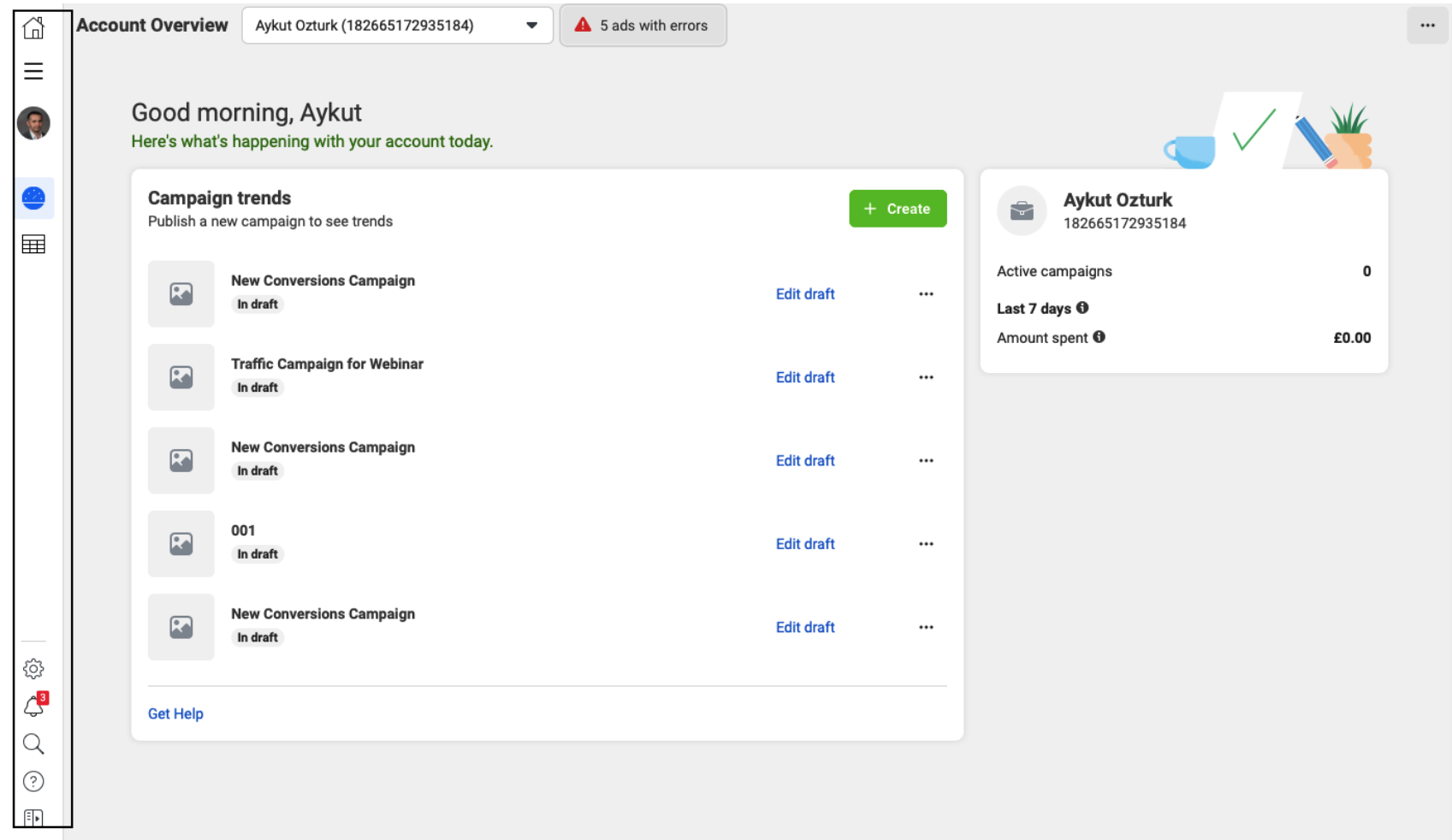

The Facebook Ads Manager is the website you will need to visit whenever you want to create a new advertisement campaign. ${ }^{3}$ In Figure Figure 3, you see the account overview page of Facebok Ads Manager, which lists all the advertisement campaigns created by your account. On the left-hand side of the screen, you see a menu, which is encircled in a black box by us. This menu allows access to other pages that will be useful for you. We will discuss the design of Facebook Ads Manager in more detail in Section 4.

\footnotetext{
${ }^{3}$ It is hosted on facebook.com/business/tools/ads-manager.
} 
If you will not be conducting conversion campaigns, the screen in Figure 3 will be enough for you. Otherwise, you will also need to visit Events Manager. You can reach Events Manager by clicking on the icon with three horizontal lines in Facebook Ads Manager, as seen in Figure 4.

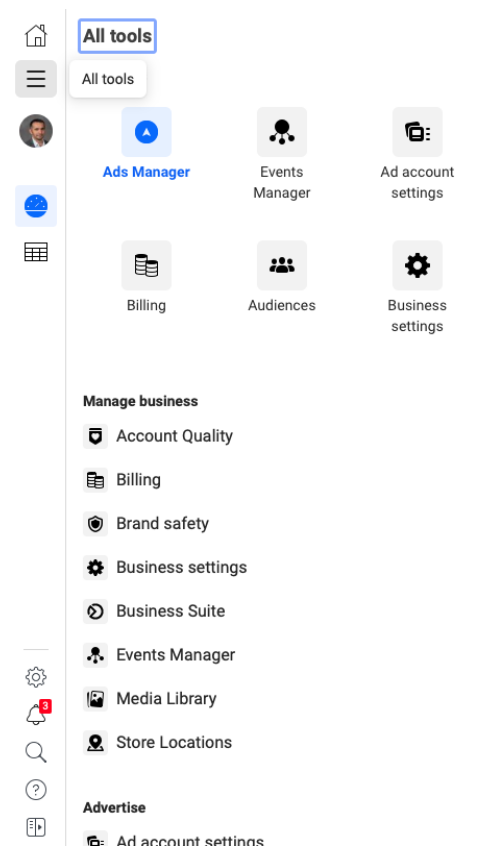

Figure 4: Visiting Events Manager

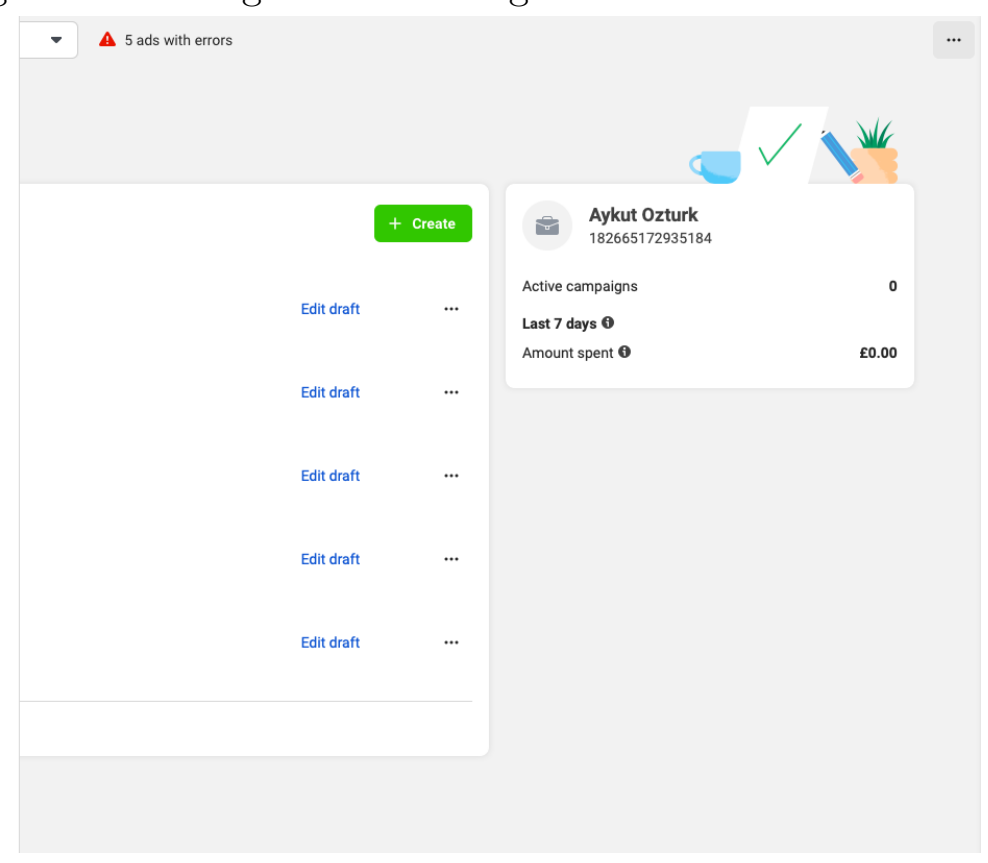

A screenshot of the Events Manager is also shown in Figure 5. "Event" refers to user behaviors that are taken outside of Facebook environment, such as the purchase of an item from your web page or completing a survey. Through Events Manager, you will be able to define user behaviors outside of the Facebook environment that will be tracked for your advertisement campaigns. You will then be able to track these behaviors in real time through the main screen on Figure 5. Again, take note of the menu on the left of the screen, encircled with a black box by us to highlight it. You will frequently use this menu, as we will detail below.

To use these web pages, you first need to have a personal Facebook account and

\footnotetext{
${ }^{4}$ If you have not yet created your Facebook Pixel, the middle of your screen will look slightly different.
} 
Figure 5: Events Manager

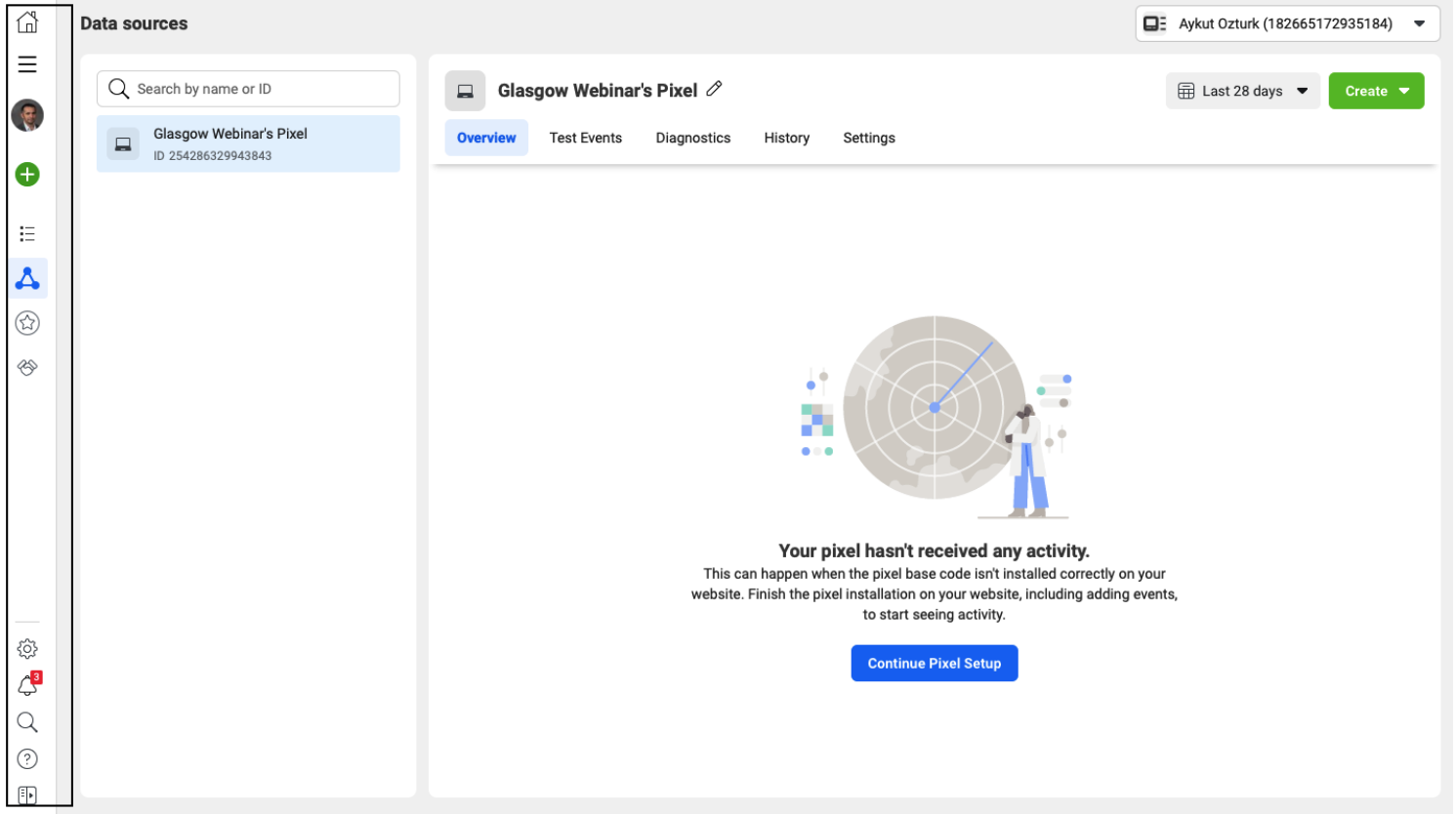

log into your account. If you create a new Facebook account and immediately attempt to create advertisements, Facebook might ask you to confirm your identity first. You will then need to upload the image of your identity card and enter your phone number. It is important to note that Facebook users, to whom your advertisements will be shown, will not see your personal Facebook profile.

Secondly, you need a Facebook page to advertise on Facebook. As shown in Figure 6 , the name and logo of your page will be located at the top of your advertisement, and Facebook users who click on them will be transferred to your Facebook page. The advertisement itself, however, will not be automatically posted on your page.

If you don't have a Facebook page, Facebook will ask you to create one once you visit the Facebook Advertisement Manager, also offering you an easy set-up tool. Simply choosing a name and a profile photo will be enough to create your first Facebook page. Since Facebook users might scroll through your Facebook page to evaluate the credibility of your advertisements, you might want to include some additional information and posts 
Figure 6: An example of Facebook advertisements

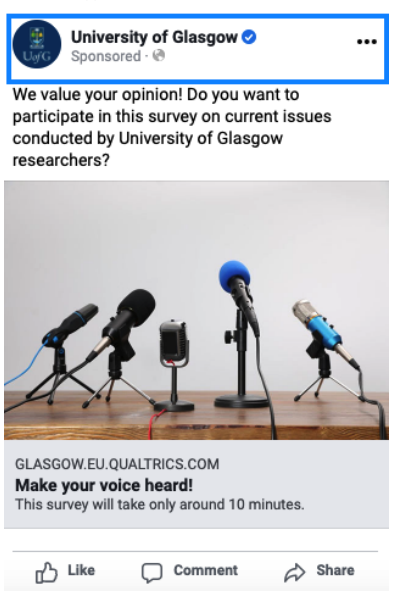

on this page.

An alternative to using your own Facebook page is to use the Facebook page of an institution that you are affiliated with. For example, you might contact your university's marketing department to see whether they will allow you to advertise through the university page. Remember that your advertisements will not be posted on the Facebook page: You are only asking permission to use the university logo and name in your advertisements. You should however bear in mind that using an institutional Facebook page might impact your credibility, in positive or negative ways, depending on which country you are advertising in.

Finally, creating a Facebook business account might be needed for some of the shortcuts listed below. You can create a business account through business.facebook.com/ create. You will need to click on "Create Account" button and enter the relevant information. However, if your account is new or not used at all, Facebook might require additional identity checks for your account. 


\section{Setting-up a conversion infrastructure}

To create a conversion campaign, you should first set-up the infrastructure that will facilitate the data transfer between Facebook and your web page. This process may take up to an hour. Once the conversion infrastructure is set-up, you can use the same infrastructure whenever you want to create a new advertisement campaign on Facebook.

Exact steps that you need to take to set-up this infrastructure will depend on how you will design your conversion flow. The flow of our conversion campaigns, which aimed to recruit participants into a Qualtrics survey, can be summarized in five steps:

1. Facebook users see our advertisement on Facebook.

2. If a Facebook user clicks on our advertisement, she is directed to our survey page hosted on Qualtrics.

3. If the Facebook user completes the survey on Qualtrics, she is directed to a "Thank You page" hosted on our Wordpress website.

4. Our Wordpress web page records this page visit as an "event" and sends information to our Facebook account about this event.

5. Our Facebook account optimizes its ad delivery based on this information.

Researchers can make some changes to this flow. Most importantly, they can avoid using a third website (i.e. Wordpress website in our case), simply using Qualtrics to record user behavior. This might seem like the most intuitive choice as researchers would not then need to direct survey takers to a third website. Using Qualtrics, however, creates several limitations. First, it requires some, although minimal, knowledge of coding. ${ }^{5}$ Second, distinguishing respondents who completed the survey from respondents who

\footnotetext{
${ }^{5}$ Our handbook covers the methods we are using in our own studies. If you want to use Qualtrics instead, some instructions are provided here: https://annie-y-chen.com/post/ setting-up-facebook-pixel-qualtrics/
} 
left the survey before completion will be easier if you use a third website.

Finally, and most importantly, as a result of Apple's most recent policies, Facebook advertisers now need to verify the ownership of their website's domain to deliver their advertisements to iOS users with the best performance. ${ }^{6}$ This is easy to do if you own the domain but we do not know whether it is possible at all with Qualtrics. If you are conducting your survey in a country in which very few people are using iOS phones, of course, this might be a small concern for you. ${ }^{7}$

Setting-up the infrastructure that underlies a conversion flow like ours takes five steps: Creating a Facebook Pixel, installing this Pixel to your website, defining an event, verifying your domain, and prioritizing your events. The first three steps are necessary, while the last two steps are recommended by Facebook to improve the delivery of your advertisements to Apple iOS users, who opted out of tracking - see Footnote 6.

Facebook usually offers more than one path to complete these five steps. There is always a standard way to do it using the menu and sections provided in the Events Manager. Alternatively, Facebook offers easy set-up tools in Facebook Ads Manager that become available if you start creating an advertisement campaign without completing these steps. These easy set-up tools can take you through the entire process of creating a conversion infrastructure. Below we will cover these multiple paths for each steps.

\footnotetext{
${ }^{6}$ As part of its policy of "protecting customer privacy", Apple has recently started to force apps to receive permission from Apple users to be able to track them. This means that Apple users can now easily opt out of tracking, which makes conversion campaigns less effective. Early data shows that most of Apple users actually opt out of tracking when this option is offered. To provide tracking data under these circumstances, Facebook offers Aggregate Event Measurement protocol. This protocol allows registering up to eight events on a domain that has already been verified by Facebook to be owned by the advertiser. More information is available here: https://www.facebook.com/business/ help/721422165168355?id=1877298665783613

${ }^{7}$ Buying a new domain and creating a "Thank You" page on a new website can take around 1 hour and cost around £30 annually.
} 


\subsection{Creating a Facebook Pixel}

Facebook Pixel is a code that is unique to an advertisement account and is used to transfer visitor data from another website to Facebook through browser cookies. It is necessary to create a Facebook Pixel code and install it to the website, in which an user actions (or as Facebook calls this, an "Event") will be tracked.

Creating a Facebook Pixel code is straightforward. You can go to Events Manager, as seen in Figure 5. On this screen, you should click on the "+" sign located at the left-hand side of the screen; you will also see a "Connect Data Sources" headline once you bring your cursor on the "+" sign. In the pop-up window that will now be opened, as seen in Figure 7, you should select "Web" and then select "Facebook Pixel." Finally, click "Connect." You will only need to name your Pixel now. ${ }^{8}$

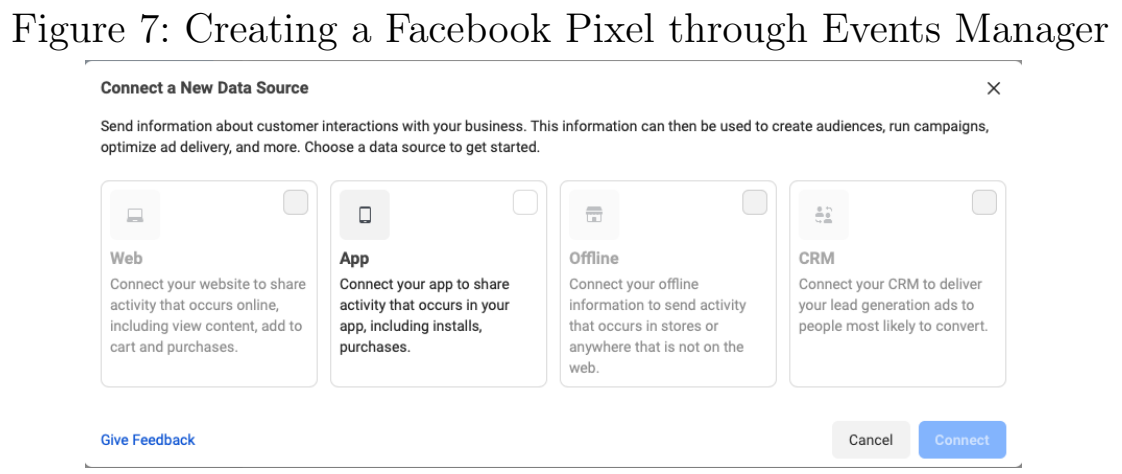

Alternatively, Facebook offers a shortcut that is available through the Facebook Ads Manager. In this case you will need to go to the Facebook Ads Manager, as seen in Figure 3, click on the green "Create" button, choose Conversion as your campaign objective, enter a campaign name, and click "Next". You have actually started creating your conversion campaign, but you do not have a Facebook Pixel yet. For this reason, you will see the "Create a Pixel" button as seen on the left-hand side of Figure 8. Once you click on it, an easy set-up tool will be opened, as it can be seen on the right-hand

\footnotetext{
${ }^{8}$ Web option in Figure 7 looks faint because we had already created a Facebook Pixel for this account. This should not be the case for you if you never created a Pixel before.
} 
side of Figure 8. First, name your Pixel. If you also enter your website's name to the box below, you will be able to continue to the second step, "Installing Facebook Pixel to your website" with this set-up tool.

Figure 8: Creating a Facebook Pixel
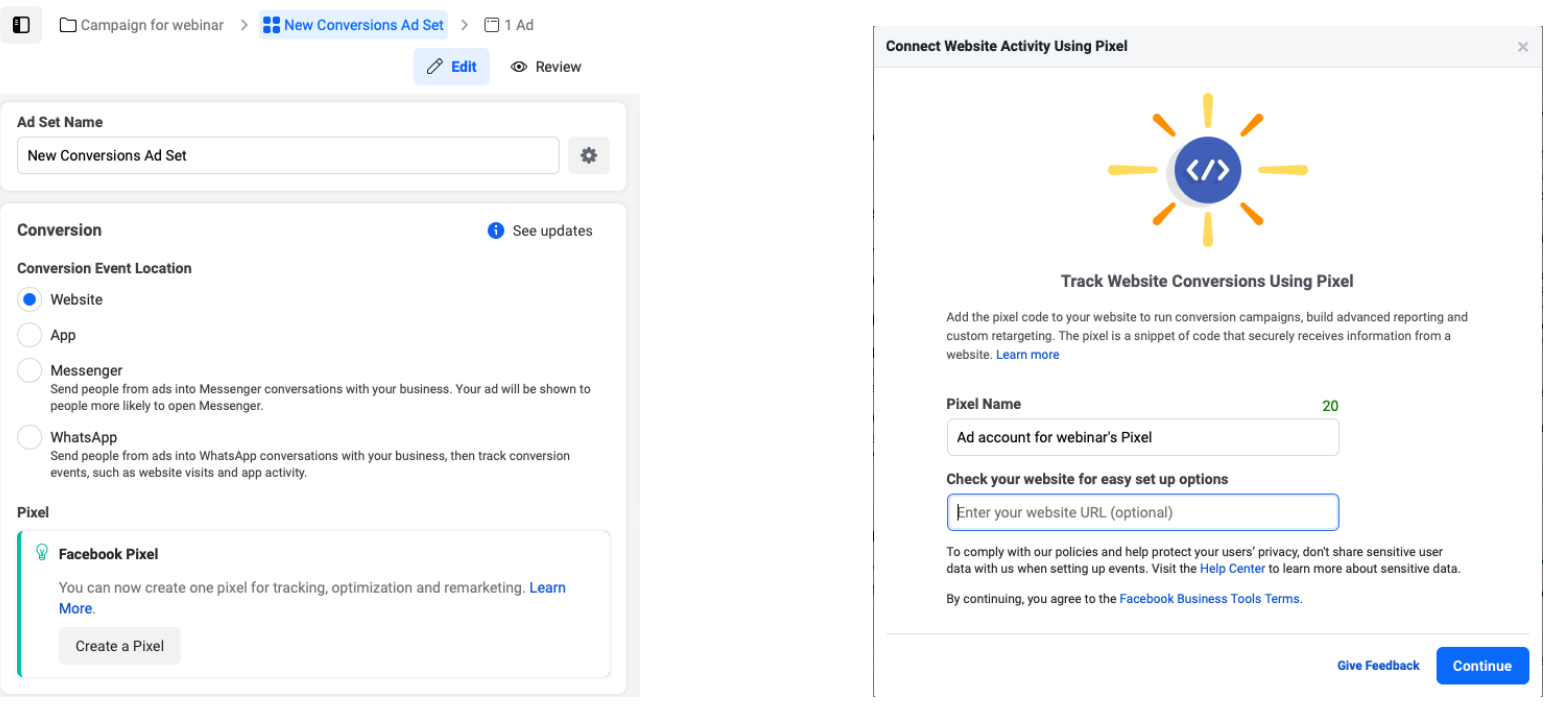

\subsection{Installing Facebook Pixel to your website}

Once a Facebook Pixel is created, you need to place this code on your website, in which the user behavior will be tracked. As explained above, this can be a website owned by you or an online survey page hosted on Qualtrics or another survey platform.

There are different ways to start the process. First, you can simply follow the easy set-up tool that is opened by you, as seen in Figure 8. Alternatively, you can again visit the Events Manager, as seen in Figure 5. On this screen, you can click on "Continue Pixel Setup" button in the middle of the page. This will open the pop-up menu, as seen in Figure 9. If you want to install your pixel manually, you should click on "manually add pixel code to website". You will need to use this option if you will install your Pixel to Qualtrics - see Footnote 5. If you click on the right button, "use a partner integration", a long list of easy set-up tools developed for commonly used web platforms, including 
Wix, Squarespace, and Wordpress, will be opened for you. ${ }^{9}$ You can see this list in Figure 10.

Figure 9: Installing Pixel on your website: alternatives

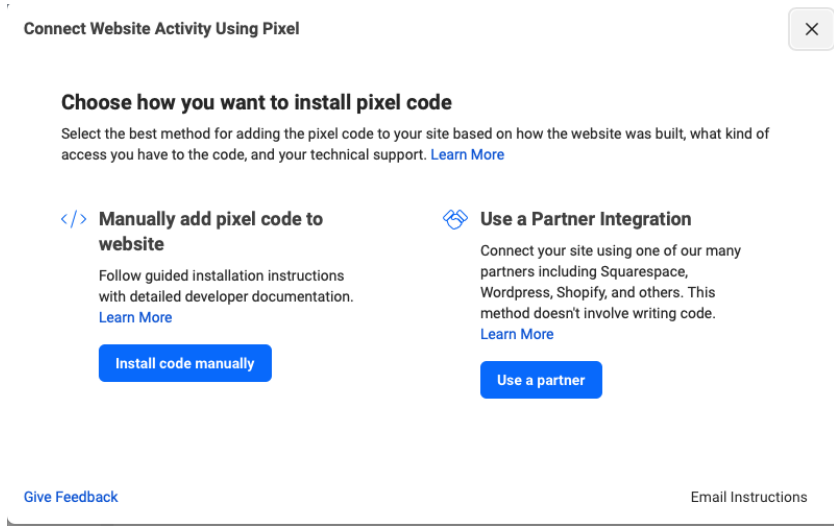

Figure 10: List of available partner integrations

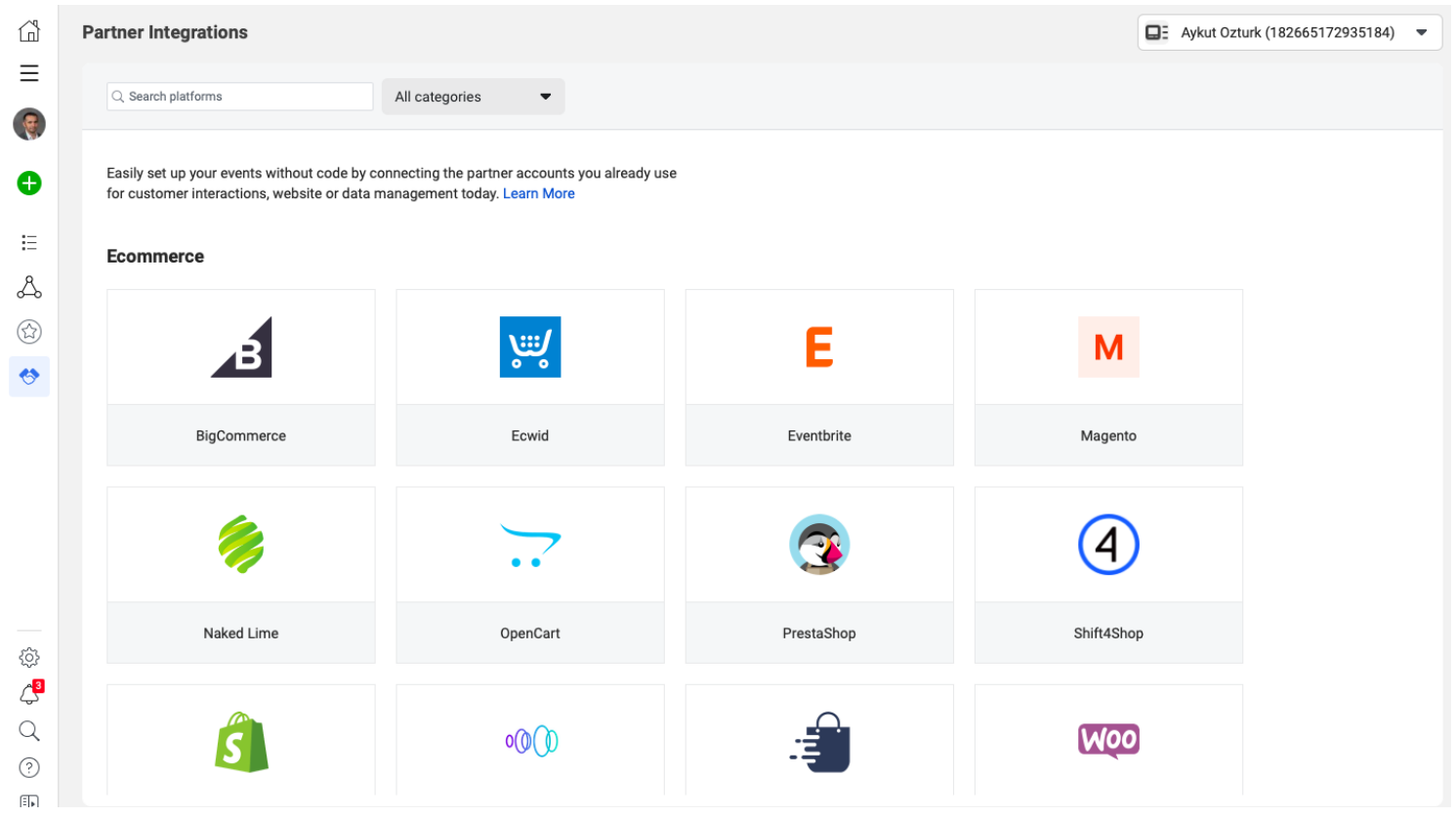

In our own study, we used a web page hosted on Wordpress. If you also choose Wordpress from the list of Figure 10, the pop-up window that is seen in Figure 11 will be opened for you. Facebook now asks you to go to your Wordpress page and install a

\footnotetext{
${ }^{9}$ You can reach this same page by clicking on the icon of handshaking, located at the left-hand side of the screen in Figure 5 You will see "Partner Integrations" headline once you bring your cursor on this icon. Once you click on this icon, you will be directed to the screen in Figure 10.
} 
plugin, named "Facebook for Wordpress." After installing this plugin, you can simply follow the instructions provided in the pop-up window to link your Wordpress page to the specific ad account and Facebook pixel that you will be using for your advertisement campaigns. Once you complete instructions on Wordpress, you can return back to the pop-up window on Facebook, as seen in Figure 11 to continue to Step 3.

Figure 11: Instructions provided by Facebook for Wordpress integration

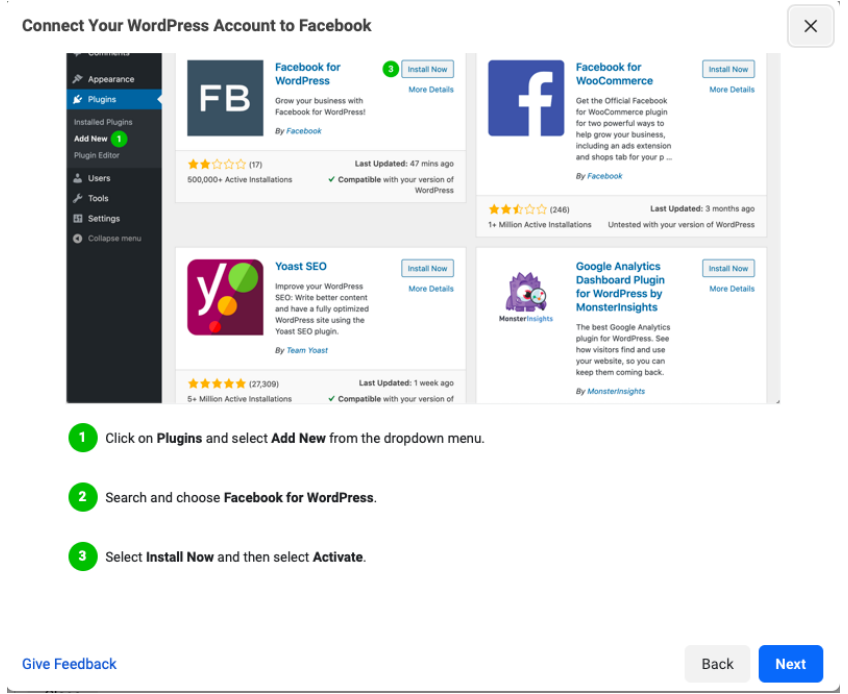

Another way to start the process of installing your Facebook Pixel your website is through Facebook Ads Manager. If you have started creating an advertisement campaign on Ads Manager and you have already created a Pixel but not yet installed it on a website, you will see the warning in Figure 12.

Figure 12: Warning to install Pixel

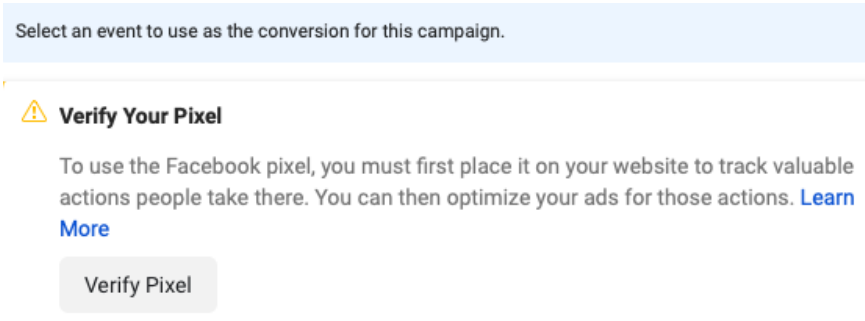

If you click on the "Verify Pixel" button to start the process of installment, Facebook 
will then list three alternative ways to add the Pixel to your website, as seen in Figure 13.

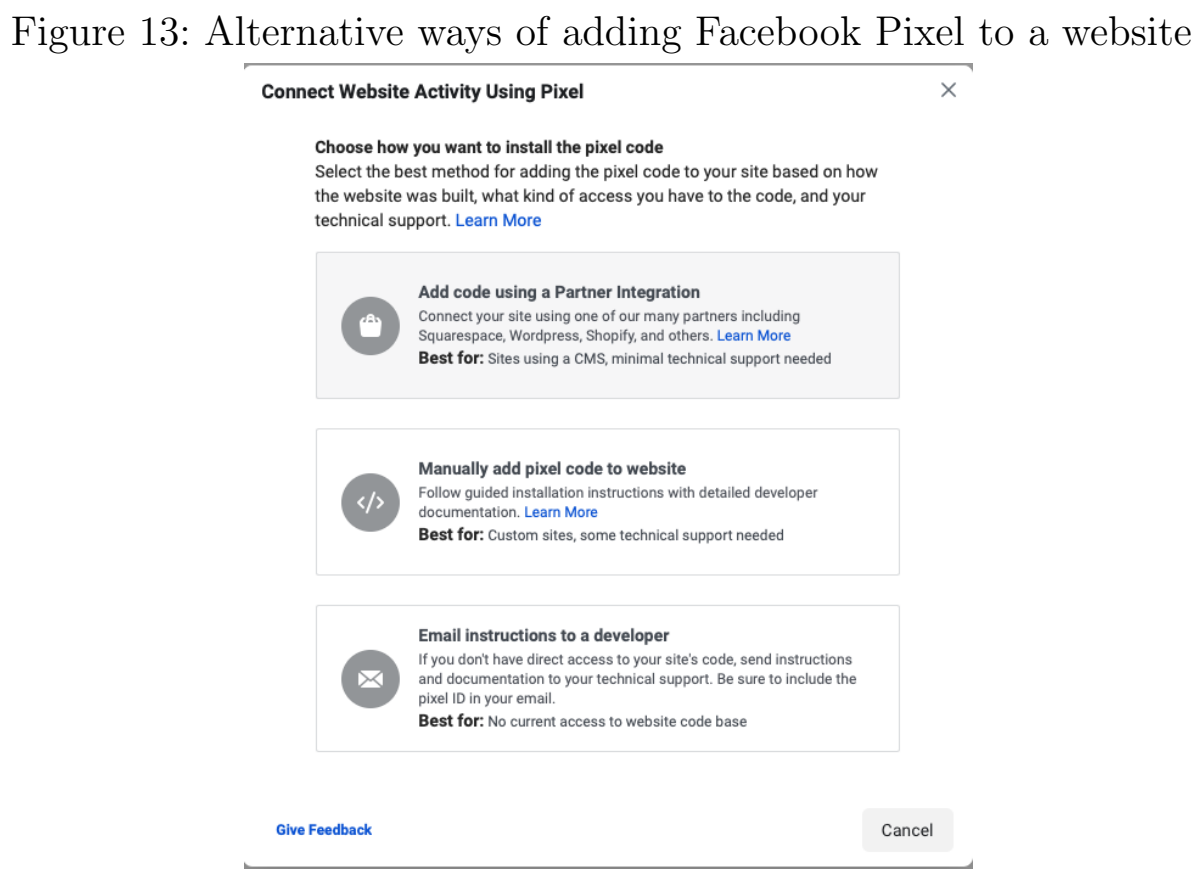

If you choose "use a partner integration," you will be directed to a pop-up window similar to Figure 10. If you want to use Qualtrics, on the other hand, you should choose the second option and enter the code yourself - see Footnote 5.

\subsection{Defining an event}

Events are actions taken by Facebook users on your website, such as a purchase, leaving contact information, or completing a survey. If you want Facebook to offer optimization towards any such events, you should first set-up that event for your website. An advertiser can set-up one of the eight standard event types that are already listed by Facebook or set-up a new event type. In our case, we set-up the standard "View Content" event as it was enough for us that our survey takers were directed to our Wordpress page after completing the questionnaire. 
As usual, you can set-up your events simply following the easy set-up tool that is opened for you in Facebook Ads Manager, as shown in Figure 14.

Figure 14: Access to event set-up tool

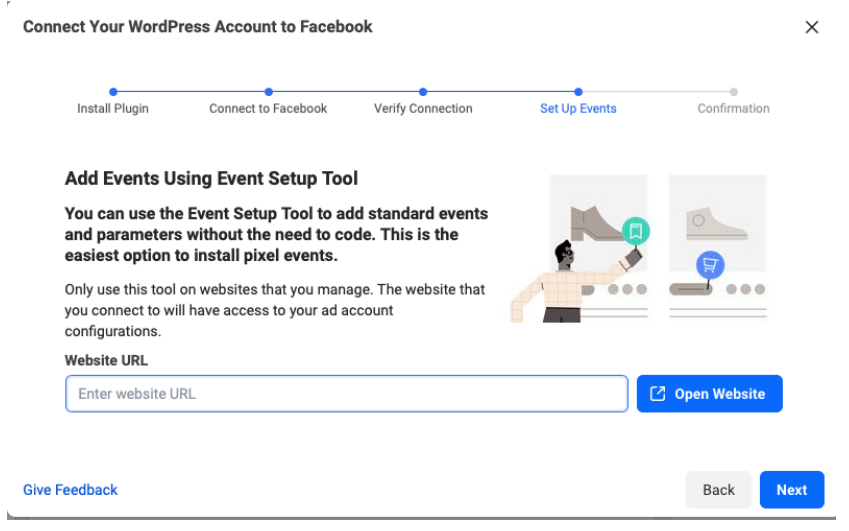

Alternatively, you can reach the event set-up tool by visiting the Events Manager, clicking on "choose a pixel" button, clicking on "settings", and choosing "event setup". In both cases, you will eventually see the box in Figure 15.

Figure 15: Access to event set-up tool through Events Manager

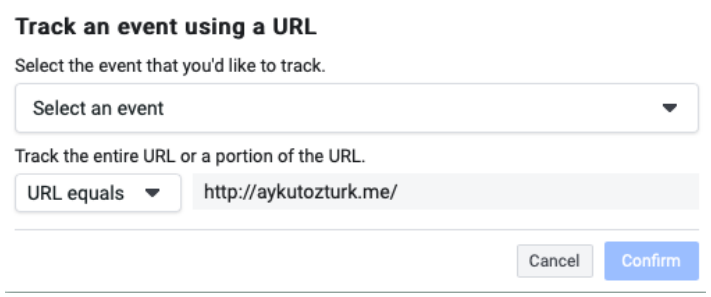

What you need to do in Figure 15 is to choose "View Content" as the event type, tell Facebook that you will track an URL, and then enter the URL of your "Thank You" page, as seen in Figure 15. You will finally need to click "Finish setup".

\subsection{Verifing domain ownership of your website}

By the end of the third step, you can start creating conversion campaigns. However, you will also receive a warning from Facebook, as seen in Figure 16, when you are choosing 
the conversion event for your advertisement campaign.

Figure 16: Warning to verify your domain

Conversion Event
View Content|
$\begin{aligned} & \text { This event hasn't been prioritized for iOS 14.5. Ad sets using this event may not be } \\ & \text { delivered to people who opt out of tracking on iOS } 14.5 \text { or later devices. Learn More }\end{aligned}$

In order to verify your domain, you can use the Events Manager. As you see in Figure 5, "Aggregated Event Measurement" section is available at the bottom half of the page. Once you click here, you can then choose "Configure Web Events" and "Manage Domains".

Figure 17: Three options to verify your domain

\begin{tabular}{|l|l|}
\hline Domains & $\begin{array}{l}\text { exampledomain.com Not Verified } \\
\text { Owned by: Siyasi Arastirmalar ID: 412821293826390 }\end{array}$ \\
\hline $\begin{array}{l}\text { Filter by name or ID } \\
\text { exampledomain.com } \\
\text { Not Verified } \\
\text { aykutozturk.me } \\
\text { Verified }\end{array}$ & \begin{tabular}{|l|l|} 
Verify your domain \\
Select one option
\end{tabular} \\
\hline Update the DNS TXT record with your domain registrar \\
\hline Udd a meta-tag to your HTML source code \\
\hline
\end{tabular}

First, of course, you will need to add your domain here. Once you click on "Add," you can enter the domain of your website. After you add the domain, you will be offered three alternatives to verify it, as seen in Figure 17, with detailed list of instructions to follow afterwards. We chose updating DNS text record option, and followed instructions.

\subsection{Prioritizing your events}

After verifying your domain, you should prioritize your events on the domain to be able to use the Aggregate Event Measurement. This will tell Facebook which events on this domain should primarily be tracked through the Aggregate Event Measurement 
protocol. In order to prioritize your events, you can again visit the Events Manager, as seen on Figure 5 and choose "Aggregated Event Measurement." You will then need to click on "Configure Web Events". Here you will find the list of domains that you added in Step 4, as seen on Figure ??. If you click on the domain you want to use for your advertisement campaign, you will see the list of events currently registered under this domain. You should then click on "Manage Events," and order the priority of your events, based on their importance for you, if you have more than one. This will complete the process of setting-up conversion infrastructure for you.

Figure 18: Configure Web Events

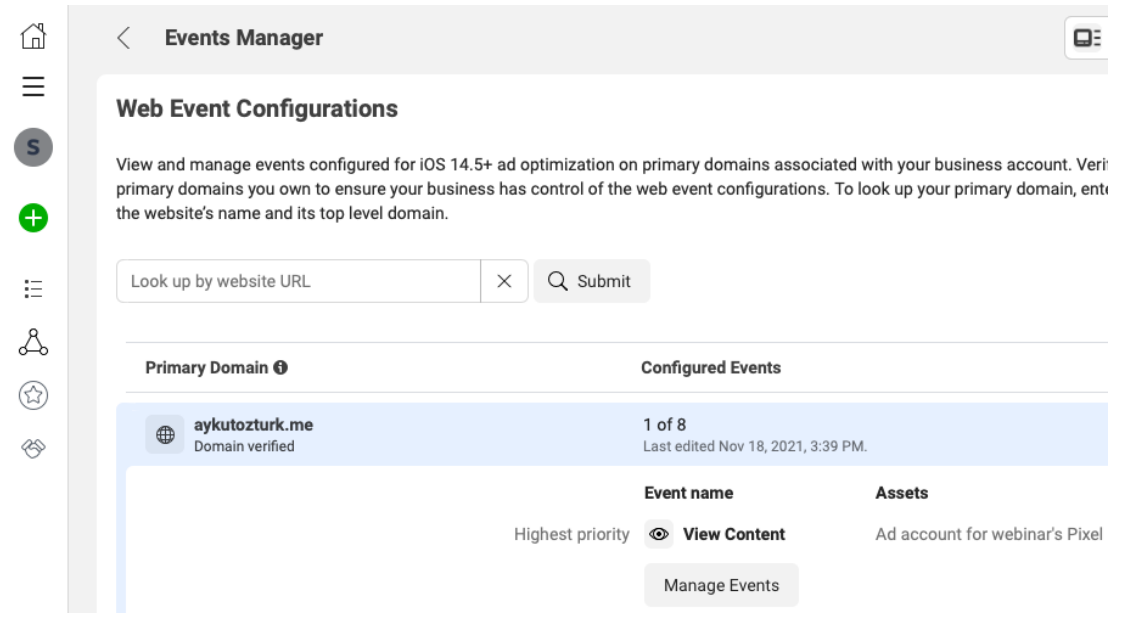




\section{Creating advertisement campaigns}

Once you set-up your conversion infrastructure, you are ready to create your first advertisement campaign. You can visit the Facebook Ads Manager and start the process. If you are planning to create traffic or reach campaigns, you do not even need to worry about setting-up a conversion infrastructure. You can immediately create your first advertisement campaign, following the steps as outlined in this section.

To create successful campaigns, and especially if you want to recruit a more representative sample through Facebook, it is important to understand the design of the Facebook Ads Manager well. In the rest of this section, we will first discuss the design of the Facebook Ads Manager. After that, we will show how you can create an advertisement campaign on Facebook. If you want to create more representative samples, you will need to add additional ad sets to your campaign, using different targets. This will be discussed afterwards. Finally, we will show how to set URL parameters of your advertisements so that you can know which advertisement each of your survey respondent saw and were recruited from.

\subsection{The design of Facebook Ads Manager}

The Facebook Ads Manager is the web page that you will be using to create actual advertisements to be run on the various Meta Inc platforms. You see a screenshot of the Account Overview page in Figure 3. You can start creating advertisements by clicking on the green "create" button, located on the left-hand side of this page. If you click on the "campaign" icon located on the left-hand menu, as encircled with a black-box, you will see the screen in Figure 19. A list of all advertisement campaigns that you created will be listed on the bottom half of this page. By using various performance metrics listed on the top half of the page, you can closely watch the performance of 
your advertisement campaigns and take action, such as changing their budget or closing them.

Figure 19: Facebook Ads Manager Campaigns section

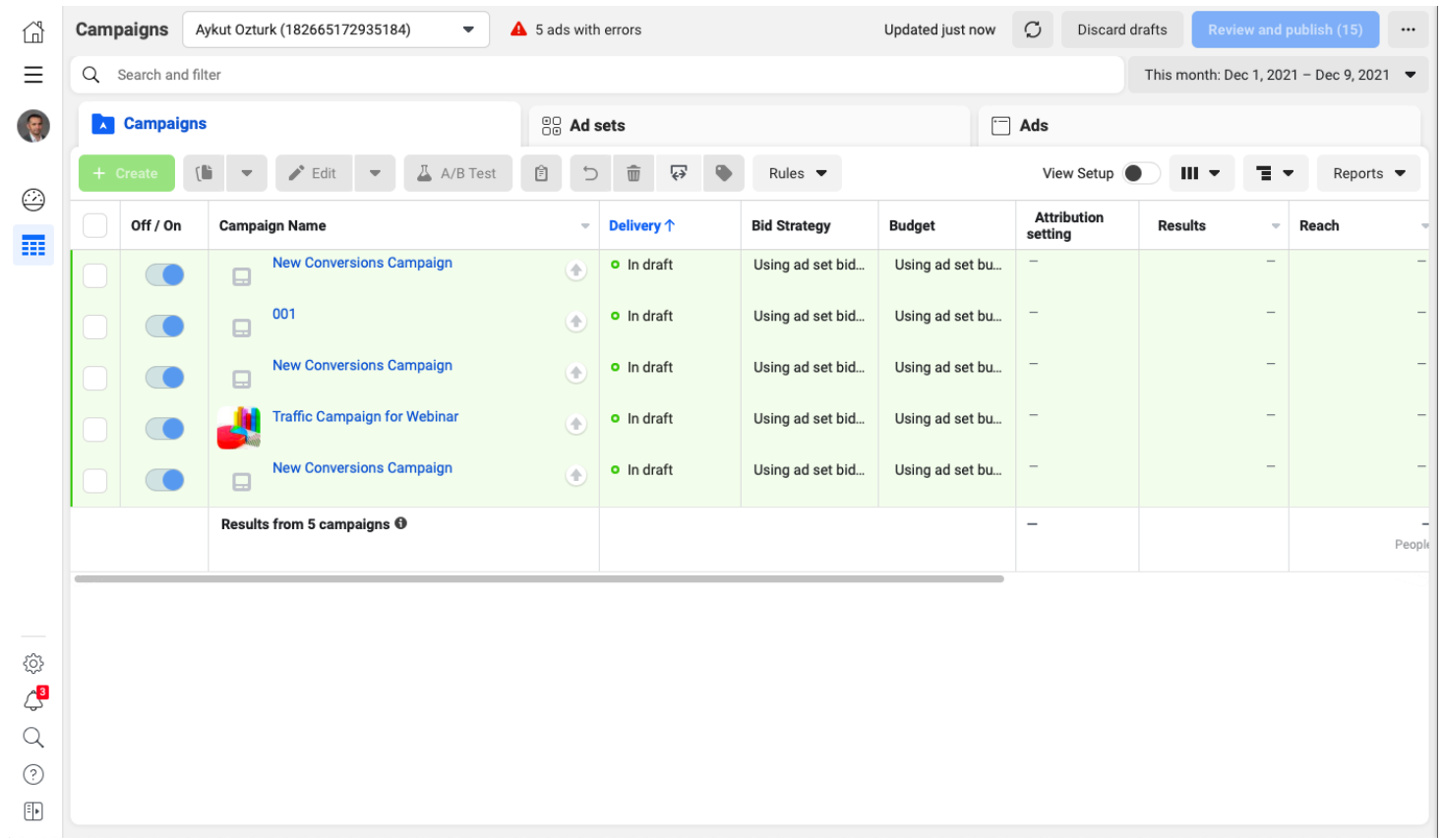

The most important thing you need to know about the Facebook Ads Manager is its hierarchical structure formed of three levels: campaign, ad set, and ad. You can see these three levels in Figure 19 too. Ad is the lowest level; this is where advertisements, as seen by Facebook users, are produced. Ad set is the medium level; each ad set can include one or more advertisements. The targeted audience of these advertisements, delivery strategies, and budgets are determined at the ad set level. Campaign is the highest level; it can include multiple ad sets, each having different audiences and advertisements. At the campaign level, advertisers choose campaign objectives, according to which Facebook offers a menu of optimization and pricing strategies. We briefly discussed the three main campaigns in Section 1.1.

As a result of this hierarchical design, a standard Facebook advertisement campaign has the structure seen in Figure 20. After creating an advertisement campaign, with 
its own objective, the advertiser can create one or more ad sets. Then, for each ad set, she creates advertisements. Each ad set should have at least one advertisement, but the advertiser can create more.

Figure 20: Structure of a standard Facebook advertisement campaign

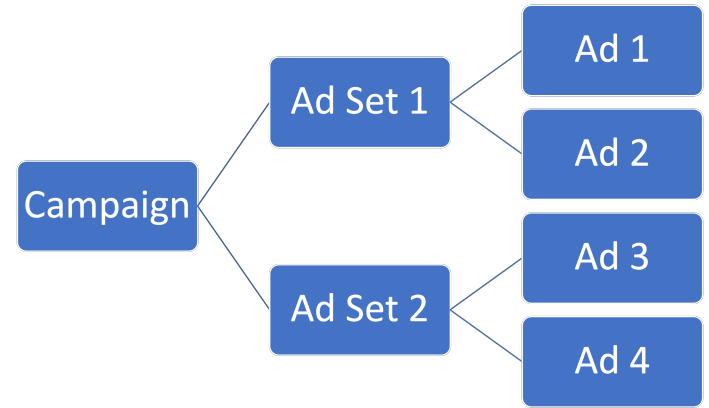

The hierarchical structure of the Facebook Ads Manager allows the use of Facebook's optimization algorithms for more cost-efficient results. By grouping advertisements under ad sets, Facebook facilitates optimization at the advertisement level. In other words, a business can prepare several different advertisements targeting the same audience and then let Facebook's optimization algorithm determine which of these advertisements works best for that target group and channel resources accordingly. The option to create multiple ad sets each having their own advertisements, on the other hand, allows to keep this process separate for separate target groups, e.g different regions, genders, or age groups.

\subsection{How to create an advertisement campaign}

To create an advertisement campaign, you should click on the green "create" button on the main page of the Facebook Ads Manager. After that, as you see in Figure 21, 
you will choose your campaign objective. ${ }^{10}$ Based on your choice, Facebook will open an easy set-up tool that will take you through each of three hierarchical levels. We picked a conversion campaign for the handbook example, but the rest is the same for all campaigns to a great extent.

Figure 21: Campaign objectives offered by Facebook

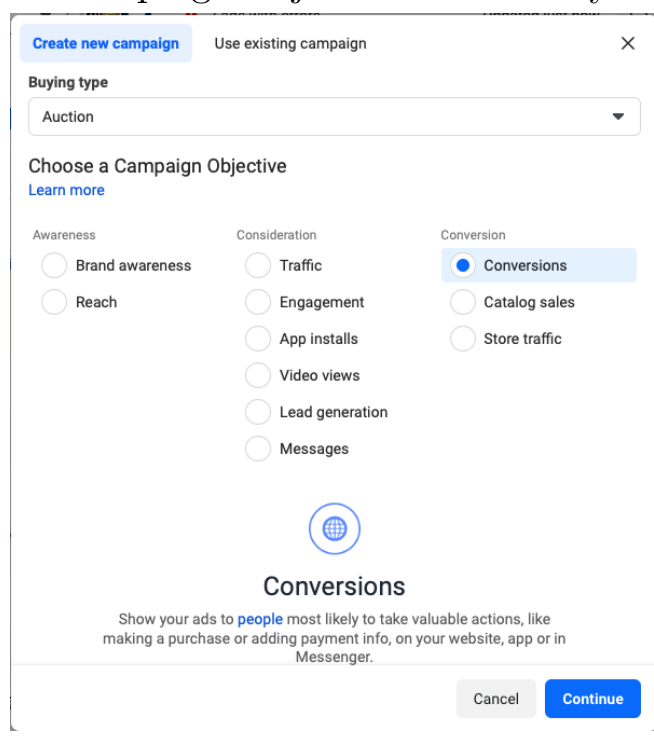

\subsubsection{Choices at campaign level}

The main screen at the campaign level, once the set-up tool is opened, can be seen at Figure 22. On the left-hand side, you can see the map of the current structure of your advertisement campaign: In this example, we have one conversion campaign that includes one ad set that includes one ad. Having the campaign highlighted with blue color indicates that you are editing that campaign. The right-hand side is empty right now; but you will see additional information here once you make edits at ad set and ad levels. Finally in the middle of the page, you see a list of decisions you need to make. You will need to scroll down this part to complete all decisions at the campaign level.

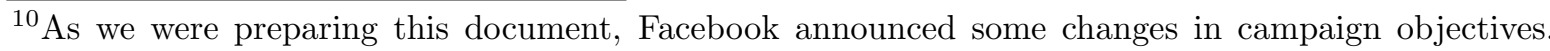
Although there will be changes in objective names, they will still have the same functions. For more information, see https://www.facebook.com/business/help/325793898950394
} 
Figure 22: Full screen of set-up tool at the campaign level
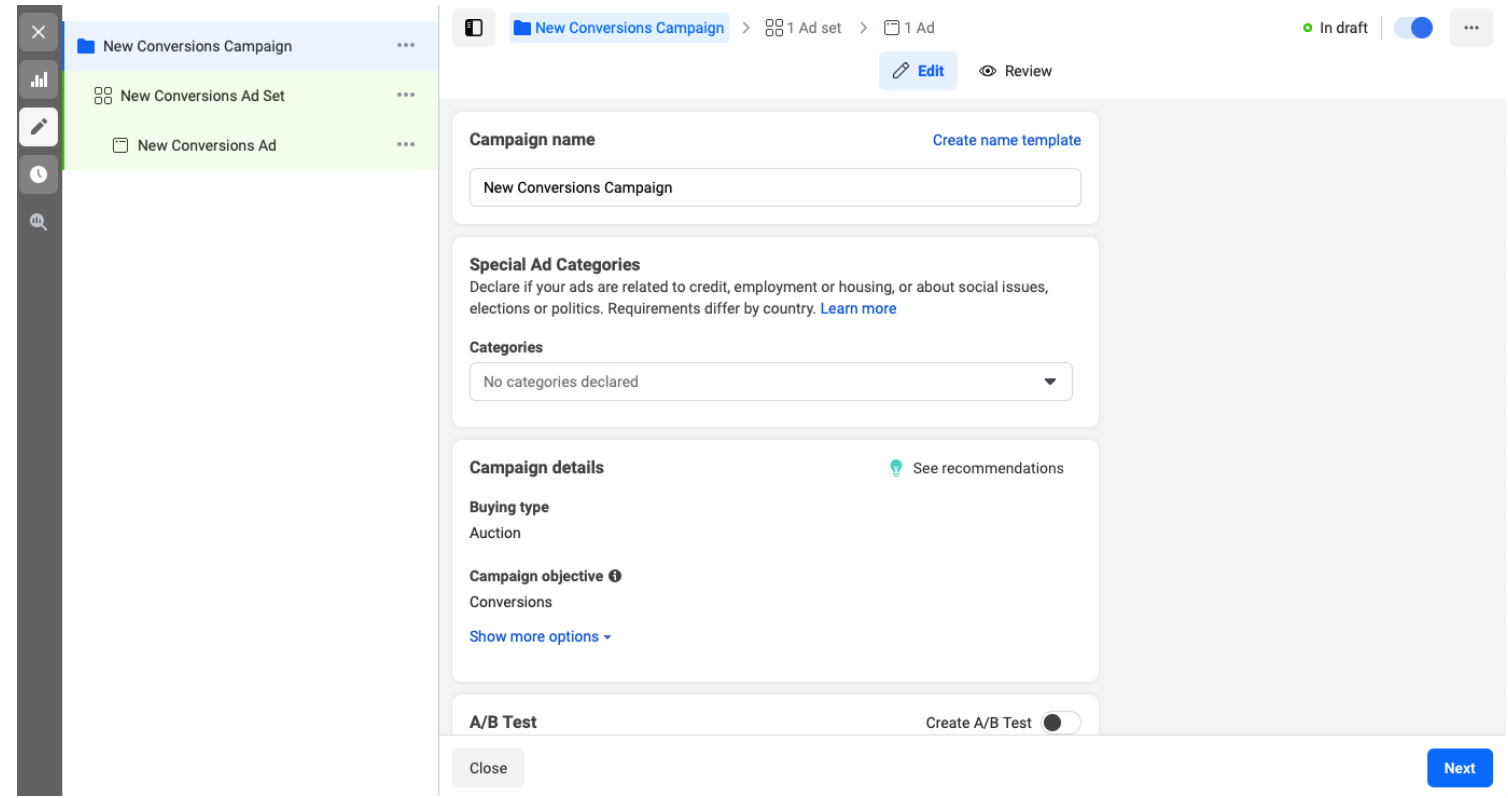

Figure 23: Special ad categories

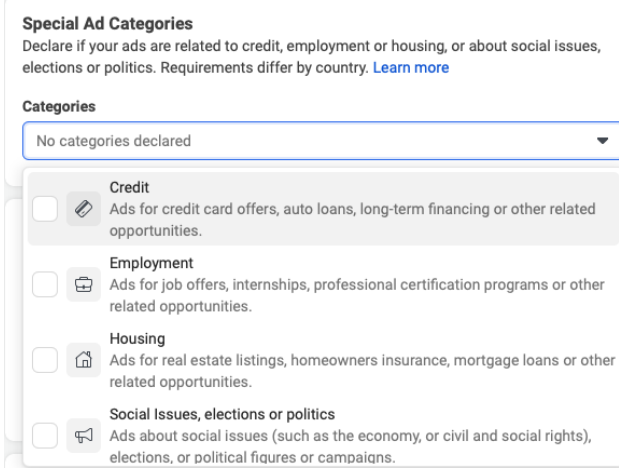

At the campaign level, one is first required to specify if an ad falls under the Special Ad Categories, as seen in Figure 23. This is required by Facebook "to enable healthy discourse and increase transparency and accountability". Advertisements falling under the special ad categories are subject to identity checks and more detailed publishing procedures. Although our survey covered political issues, our Facebook advertisements did not. Most of our advertisements did not mention politics at all. For these reasons, we did not list our advertisements under the Special Ad Categories.

There are several other tools that can be chosen at the campaign level. A/B tests 
can be used to test how variations of campaigns, ad sets, or ads work in comparison to each other. These will not be as rigorous as experiments, however. You can also set a spending limit at the campaign level; but we always set our spending limits at the ad set level.

\subsubsection{Choices at ad set level}

Once you complete your choices at the campaign level and click the "Next" button, you are directed to the ad set level. The full screen of the set-up tool at the ad set level is visible in Figure 29.

Figure 24: Full screen of the set-up tool at the ad set level

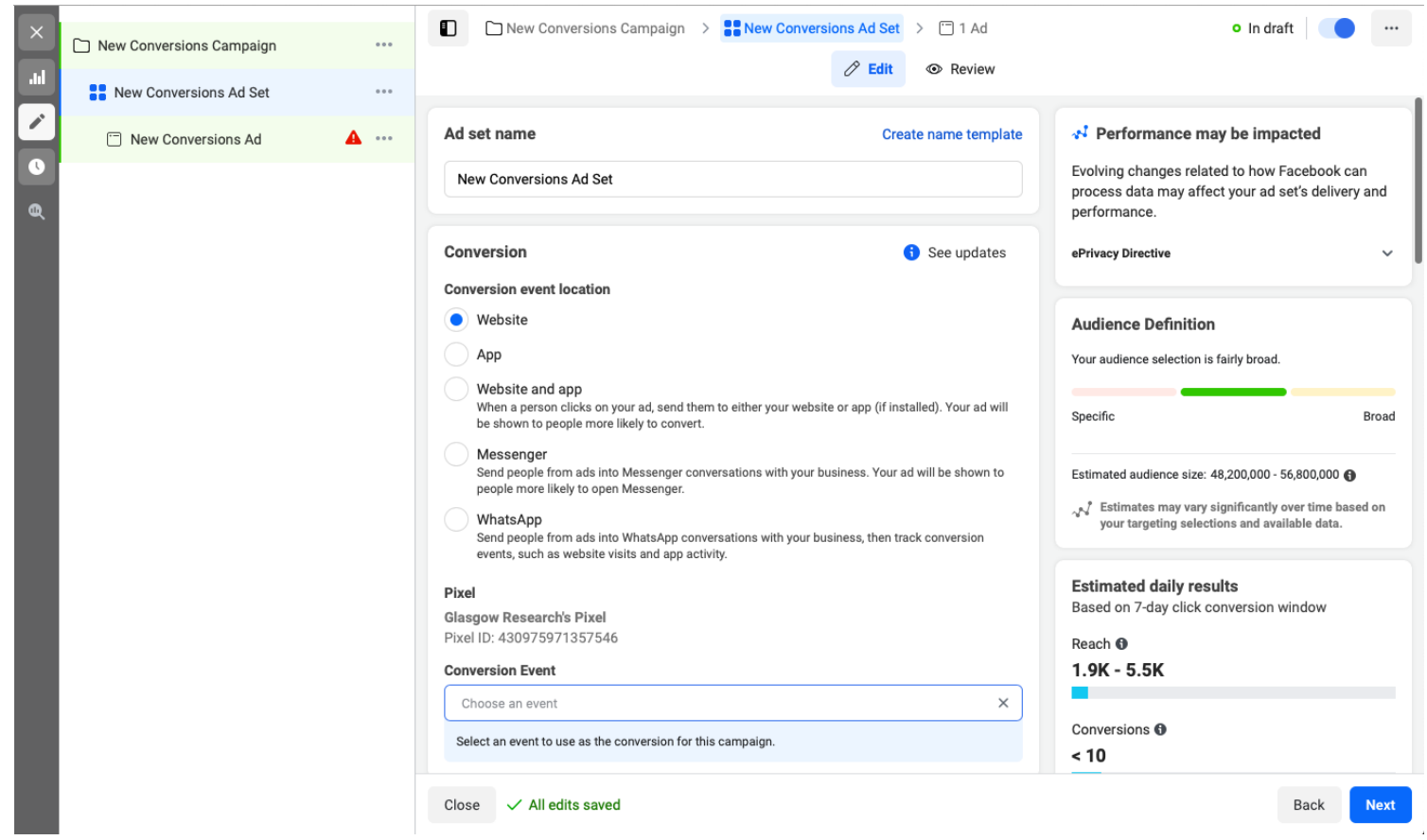

On the left hand side, you again see the map of the current advertisement campaign, whereas the blue highlight shows that you are editing the ad set. On the right hand side, you see the estimated audience and daily result estimates. These estimates will change based on the decisions you make on this page. 
Figure 25: Choosing conversion event

\begin{tabular}{|c|c|}
\hline \multicolumn{2}{|l|}{ Conversion Event } \\
\hline Choose an event & $x$ \\
\hline Add to Cart & $\Delta$ \\
\hline Add to Wishlist & $\Delta$ \\
\hline Donate & $\Delta$ \\
\hline Schedule & $\Delta$ \\
\hline Subscribe & $\Delta$ \\
\hline aozturk.net jan30 & $\Delta$ \\
\hline View Content & \\
\hline
\end{tabular}

Here we see the "conversion event" section because we are creating a conversion campaign. Here we need to choose which event this ad set will optimize towards, as seen in the opening menu in Figure 25. In the previous section, we had already set-up a "View Content" event. This is why the only event without a yellow warning in this image is a "View Content". We need to choose "View Content" as the conversion event of this ad set.

Figure 26: Budget and Schedule

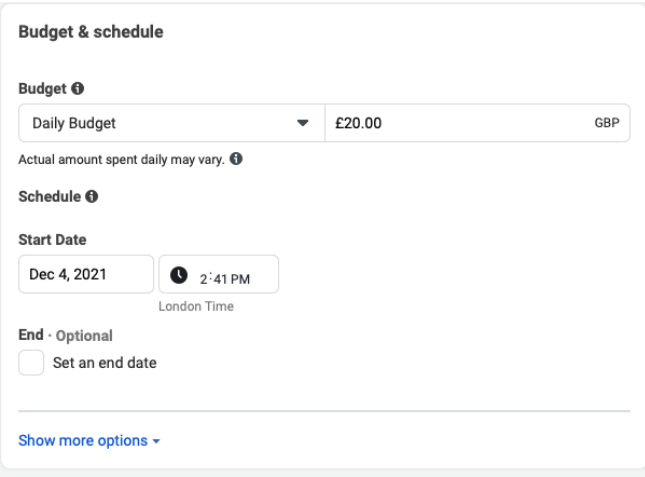

Furthermore, you can choose your budget at the ad set level or at the campaign level. We always used ad set level budgets to control how many people we would recruit with each ad set. In Figure 26 you can see the budget choices at the ad set level. For ad sets, one can either choose a daily budget or a lifetime budget. A daily budget sets a daily spending limit for Facebook. We opted for a daily budget for each ad set so that we could closely follow the performance of advertisements each day and change their 
delivery settings based on the performance. In our study, each ad set included only one advertisement so these spending limits were also spending limits at the ads level.

The audience of each advertisement is also determined at the ad set level, as seen in Figure 27. The default setting here is "create new audience"; you can also save your audience preferences to use them later through "use saved audience" setting. "Custom audience" option allows you to include or exclude people who have already interacted with your ads. You can skip this option if you are using Facebook to recruit new participants for your research.

Figure 27: Determining the audience

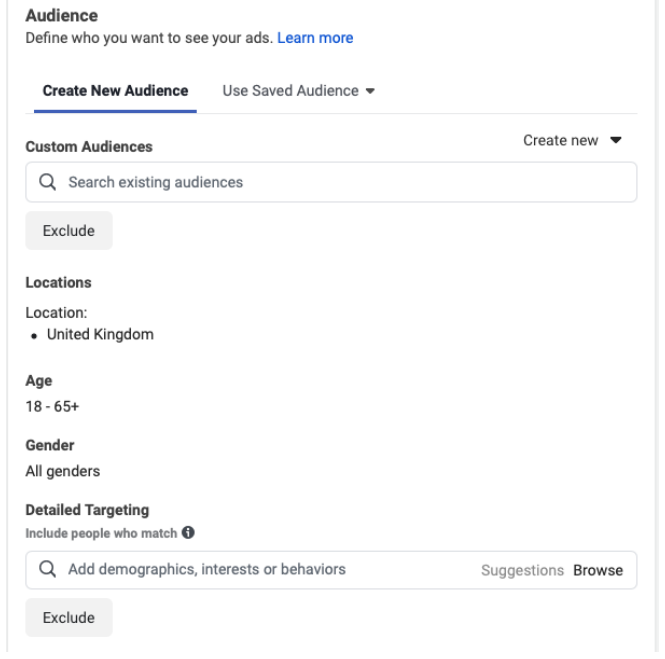

As you can see in Figure 27, Facebook offers four direct targeting categories: location, age, gender, languages. By making choices across these categories, you effectively limit the audience size of your advertisements to groups selected by you. Facebook also offers a wide range of detailed targeting options across demographics, interests, and behaviors. ${ }^{11}$ Facebook says that it will use choices across these categories as a guide, but it can still deliver your advertisement beyond those choices if that will improve the overall performance. Education level, probably most importantly for researchers, is listed here,

\footnotetext{
${ }^{11}$ These categories are built using both the data entered by the Facebook user, such as age and gender
} information, and the behavior of Facebook user on Facebook, such as likes and group memberships. 
as seen in Figure 28.

Figure 28: Detailed targeting options

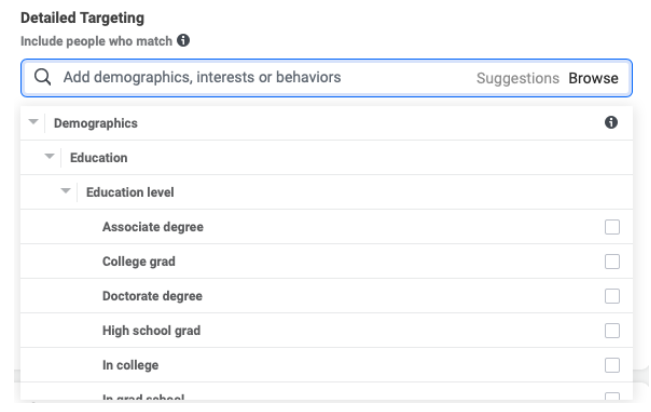

The ad set level is especially relevant for researchers if they want to create representative samples or create ads to target hard-to-reach populations, such as migrants or specific occupational groups. ${ }^{12}$ In this case, they will need to create one ad set for each of demographic categories that they want to be represented in their sample. We discuss the logic and practice of targeting for representative samples in more detail in Subsection 4.3.

\subsubsection{Choices at ad level}

In Figure 29, you see the full screen of the set-up tool at the ad level. This is where you will finally be creating the advertisement that social media users will see. On the left-hand side of the screen, you see the map of your advertisement campaign. On the right-hand side of this screen, you will see a preview of your advertisement, once you create it.

At the ad level, the middle pane is formed of seven boxes: ad name, identity, ad set-up, ad creative, destination, language, and tracking. After giving a name to your advertisement, so that you can distinguish it from other advertisements that might be

\footnotetext{
${ }^{12}$ On the topic of how to recruit research participants from hard-to-reach populations, we recommend the recording and slides of the webinar on the topic by Dr Steffen Pötzschke. These are available on our website: https://www.gla.ac.uk/research/az/democracyresearch/datamethods/ facebookasaresearchtool/.
} 
Figure 29: Full screen at ad level
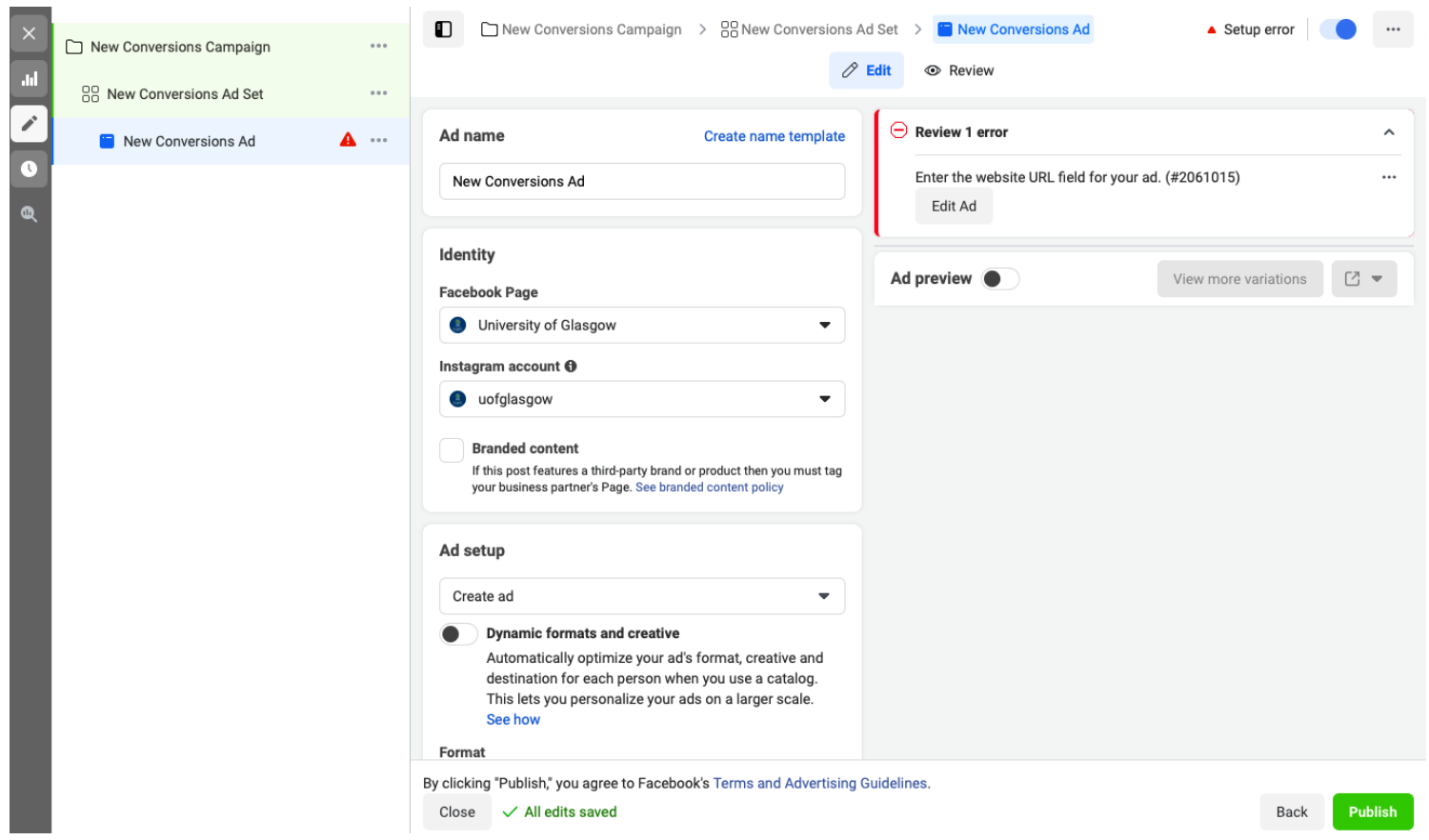

part of the same ad set, you can choose which Facebook page of yours will be used to deliver your advertisements. In the ad setup box, you should choose "create ad." We used a single image in all of our ads, but you can also try using multiple images, videos, or other options. At the ad creative box, you can finally upload the image you will be using in your advertisement and enter the primary text, headline, and description for your advertisement. At the destination box, you should enter the URL link you want to direct Facebook users at. In our case, this was the URL link of our Qualtrics survey; people who clicked on our advertisement were directed to this URL link.

The final box, tracking, allows you to follow where your survey participants are coming from. You can use this box to register which advertisement, ad set, campaign, and platform your survey participant is coming from. This is a necessary tool especially if you are planning to conduct methodological analysis; we will discuss how to do this in Subsection 4.4 below.

After completing all of these steps, clicking on "Publish" button submits the ad- 
vertisement campaign to Facebook's review, which can take from several hours to one day. Once your advertisement is online, it is advised to follow its performance and user comments through Facebook Ads Manager. ${ }^{13}$

\subsection{Using targeting tools to create more representative samples}

\subsubsection{The logic}

Despite having users across all demographic groups, Facebook samples might end up being highly skewed towards certain demographic groups, such as older people, male, and college educated people. This is a result of both the self-selection bias and the way Facebook's optimization algorithms work. If you want to have samples in which all demographic groups are represented to a certain extent, you can use Facebook's targeting tools.

As discussed above, Facebook offers a wide range of targeting options for your advertisement campaigns. These targeting choices are available at the level of ad set. Each ad set has its own target audience and each ad set has its own budget. Thus, if you create separate ad sets with separate targets, you can ensure that people from these groups will be represented in your sample.

For example, let's assume that you want to ensure that younger and older people are equally represented in your sample. In this case, you need to create two separate ad sets:

- Ad Set 1: only targets younger Facebook users; it has its own budget

- Ad Set 2: only targets older Facebook users; it has its own budget

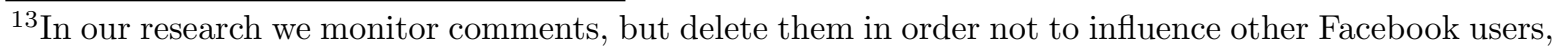
especially as our surveys usually include a survey experiment and we hence want to avoid that user comments prime potential new recruits.
} 
Because of the hierarchical structure of Facebook Ads Manager, you will need to place an advertisement under each ad set. However, you can place the same advertisement under each ad sets by simply duplicating the advertisement. The overall design of this advertisement campaign will then look like Figure 30.

Figure 30: Design of an advertisement campaign using targeting tools for representative samples

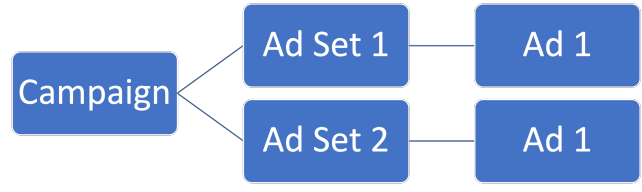

Since budget and schedule of advertisements can be determined at ad set level, you will be able to keep each ad set open until you reach the targeted participant number in each category. For example, if your goal is to reach equal number of older and younger participants and you see that a higher number of older people have participated in your survey than younger people, you can simply close Ad Set 2 while still keeping Ad Set 1 open. Alternatively, you can decrease the budget of Ad Set 2 and increase the budget of Ad Set 1.

There are a couple points to note here. First of all, as already implied, equal budget does not ensure that equal number of respondents will participate in your survey from each group. In this case, you will need to adjust your ad set budgets and schedule manually to ensure that you recruit the right number of people from each group. Secondly, Facebook does not always deliver what it promises. Age, gender, and location are more successful than other targets. And finally, using targets might increase costs. To get a better idea of how costs change in response to targeting, you can check neundorf2021recruiting. 


\subsubsection{The practice}

Continuing with the same example, let's assume that you want to create an advertisement campaign in which older and younger people are equally represented. What you need to do is to create two separate ad sets for each of these groups.

You can start following our instructions in Section 4.2 and create the first part of your advertisement campaign: a campaign, Ad Set 1 (i.e. the ad set for younger people) and an advertisement. Figure 31 shows the full screen at this stage of your campaign.

Figure 31: Design of an example advertisement campaign

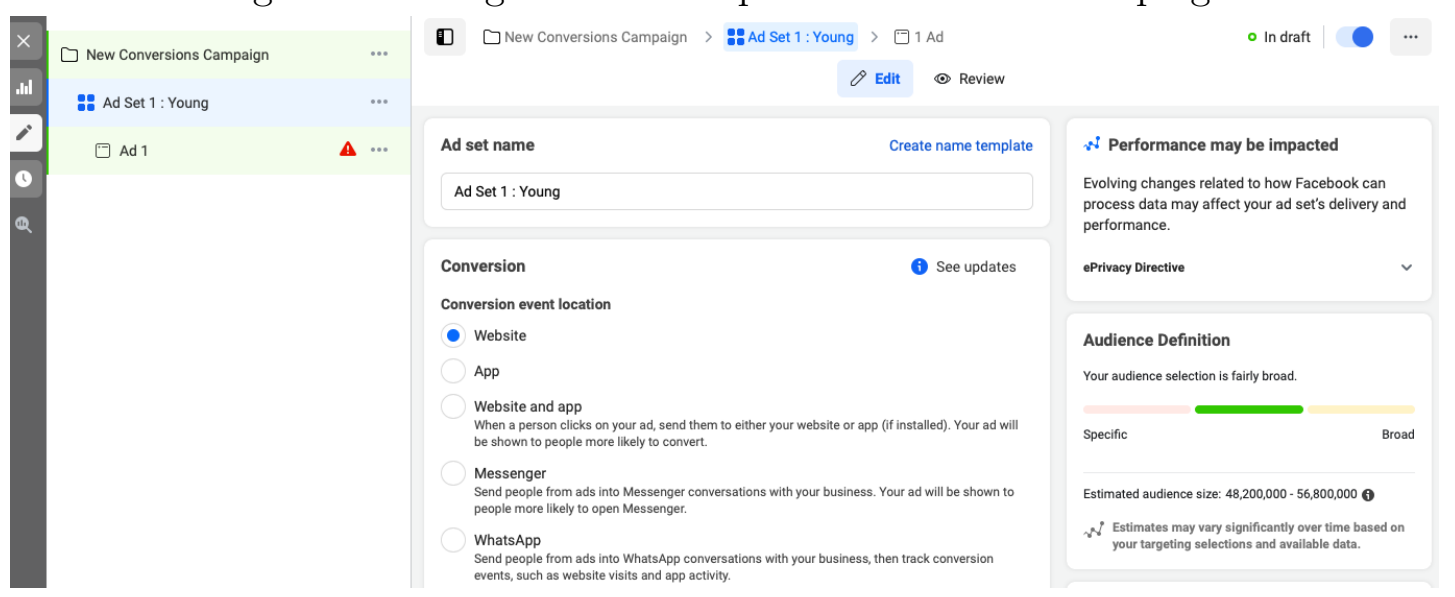

What you need to do next is to create a second ad set, grouped under the same advertisement campaign and using the same advertisement as in Ad Set 1 (targeting younger Facebook users). An easy way to do this is to duplicate Ad Set 1 and then adjust its settings. Most importantly, as you duplicate Ad Set 1, the advertisement under Ad Set 1 will also be automatically duplicated. As a result, you will not need to create the same advertisement from scratch for Ad Set 2 (targeting older users).

In order to duplicate the Ad Set 1, you should first go to the left hand side of your screen, and click on three dots placed next Ad Set 1. As seen in Figure 32, this will open an action menu for Ad Set 1. You can choose the "Quickly Duplicate" or the "Duplicate" options in this menu, depending on how many ad sets you will need. 
Figure 32: Duplicating ad set
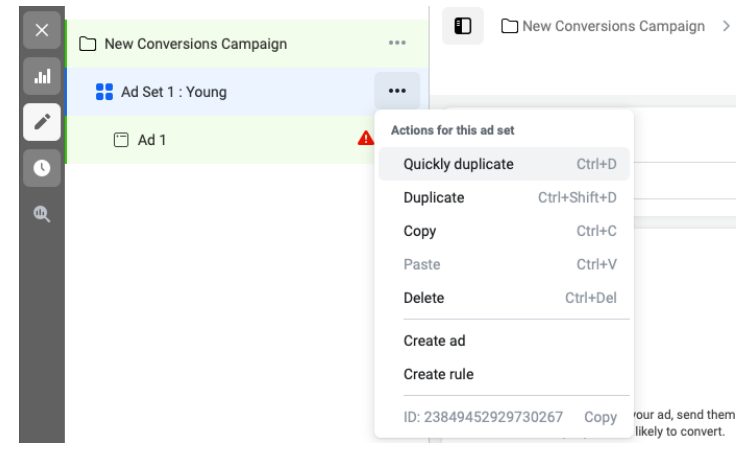

Once you duplicate Ad Set 1, your screen will look like Figure 33. What you need to do now is to go through the settings of the new ad set, i.e. "Ad Set 1 - Young - Copy" and turn this ad set into "Ad Set 2 - Old" by changing the name and targets of your ad set.

Figure 33: Main screen after duplication

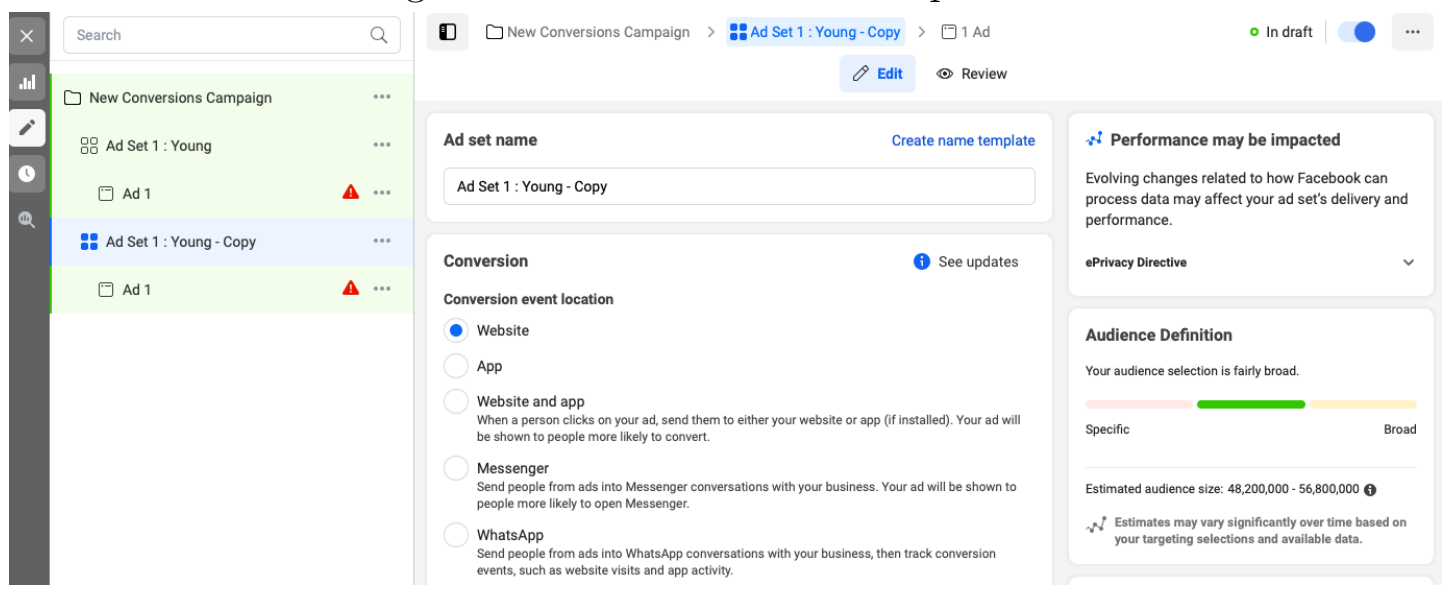

This example is, of course, significantly easier than what it would take to create more representative samples. You might want to create targets for gender, location, education, and other categories. You might also want to target intersections of subcategories, such as young woman, old woman, young man, old man. These will significantly increase the number of ad sets you will need to create. If you will be creating a very high number of ad sets and you are comfortable with coding, you might consider using the Marketing 
API to make the process easier. ${ }^{14}$ The logic, nevertheless, will always be the same.

\subsection{Setting URL parameters to know where your survey respondents are coming from}

Facebook allows you to know which advertisement, ad set, campaign, and platform your survey participant is coming from. Capturing this information is important especially if you are planning to conduct methodological analysis of your survey participants. This takes a two-steps process. First, you need to tell Facebook which information you want to be transferred from Facebook. Facebook will then add these parameters to the end of your website URL. In the second step, you need to tell Qualtrics, or your survey platform, to capture these parameters from the URL each respondent is coming from.

Figure 34: Tracking box at the ad level

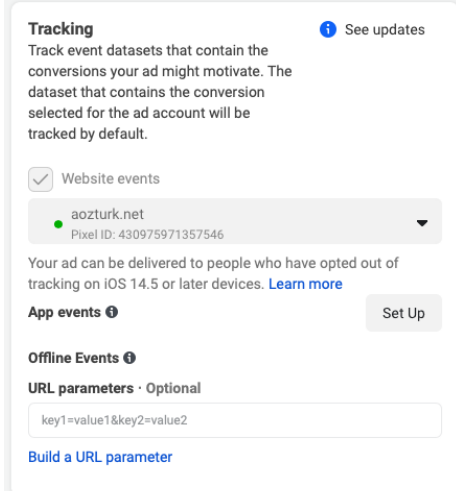

To start with, you should first go to the Tracking box located at the ad level, as seen in Figure 34. At the bottom of this box, there is an option called "Build a URL parameter." You should click on that option.

In the opening list, as seen in Figure 35, choose which parameters you want to carry to Qualtrics. You do not need to worry about parameter categories (e.g. campaign source,

\footnotetext{
${ }^{14}$ For more information, visit https://developers.facebook.com/docs/marketing-apis/get-started/
} 
campaign medium), these will only become variable names in your eventual dataset.

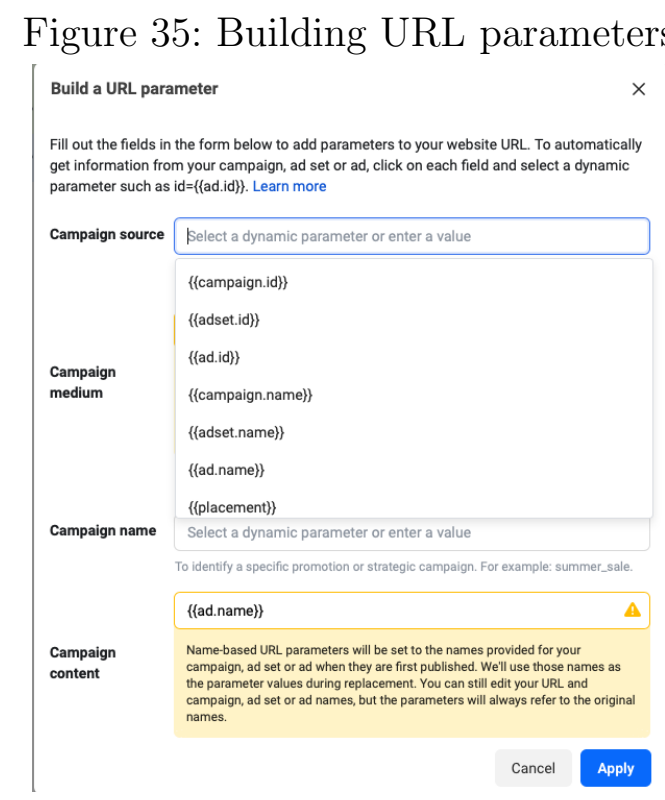

Once you choose each of your parameters, URL parameters for your survey respondents will take the form as shown in the bottom part of Figure 36.

Figure 36: Final form of URL parameters

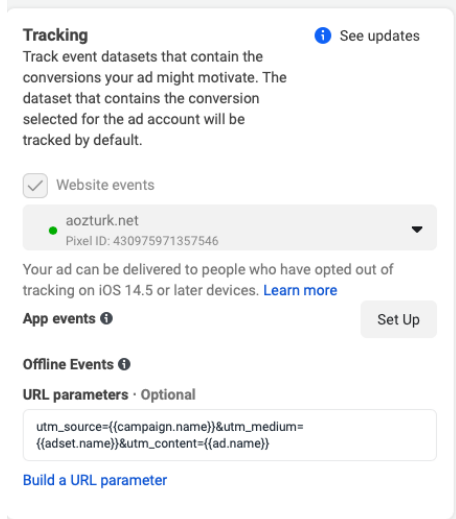

Next, you need to visit your survey platform and make sure that this data is recorded in your dataset. In the case of Qualtrics, you need to visit the survey flow section, and add an embedded data to the existing survey flow as seen in Figure 37.

On this screen, you will need to enter each of the names of the parameter categories 
Figure 37: Embedded Data on Qualtrics

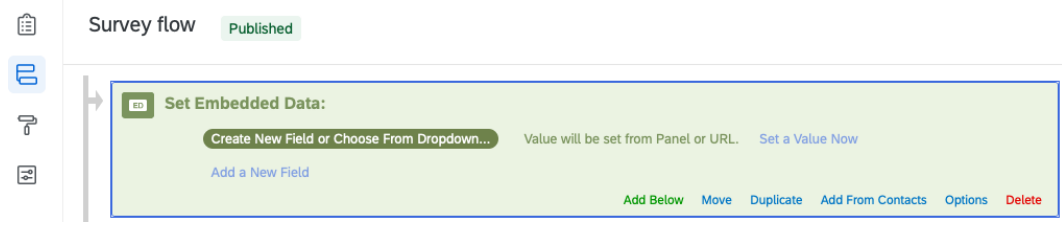

from Figure 36, such as utm_source, utm_medium etc. As values of these parameters will be set through the URL, you do not need to make any other changes here. You can see the final screen in Figure 38.

Figure 38: Setting embedded data on Qualtrics

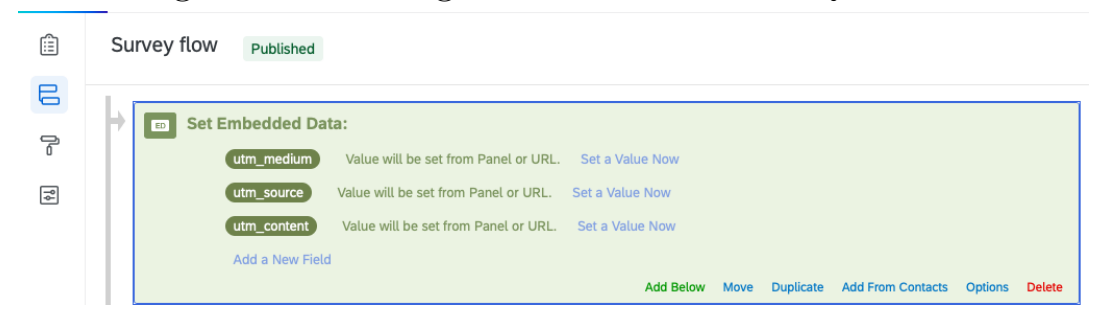

Once you complete this process, the name you gave to each of your advertisements and ad sets will be a variable in your dataset. 


\section{Bibliography}

Below is a list of papers that explore best practices to recruit survey participants through

Facebook paid advertisements. This list is not exhaustive.

Antoun, C., Zhang, C., Conrad, F. G., \& Schober, M. F. (2016). Comparisons of online recruitment strategies for convenience samples: Craigslist, Google AdWords, Facebook, and Amazon Mechanical Turk. Field Methods, 28(3), 231-246.

Boas, T. C., Christenson, D. P., \& Glick, D. M. (2020). Recruiting large online samples in the United States and India: Facebook, Mechanical Turk, and Qualtrics. Political Science Research and Methods, 8(2), 232-250.

Bunge, E. L., Taylor, L. A., Bond, M., Stephens, T. N., Nishimuta, K., Barrera, A. Z., Wickham, R., \& Muñoz, R. F. (2019). Facebook for recruiting Spanish-and Englishspeaking smokers. Internet interventions, 17, 100238.

Choi, I., Milne, D. N., Glozier, N., Peters, D., Harvey, S. B., \& Calvo, R. A. (2017). Using different Facebook advertisements to recruit men for an online mental health study: Engagement and selection bias. Internet Interventions, 8, 27-34.

Grow, A., Perrotta, D., Del Fava, E., Cimentada, J., Rampazzo, F., Gil-Clavel, S., \& Zagheni, E. (2020). Addressing public health emergencies via Facebook surveys: Advantages, challenges, and practical considerations. Journal of Medical Internet Research, 22(12), e20653.

Iannelli, L., Giglietto, F., Rossi, L., \& Zurovac, E. (2020). Facebook digital traces for survey research: Assessing the efficiency and effectiveness of a Facebook ad-based procedure for recruiting online survey respondents in niche and difficult-to-reach populations. Social Science Computer Review, 38(4), 462-476.

Jäger, K. (2017). The potential of online sampling for studying political activists around the world and across time. Political Analysis, 25(3), 329-343. 
Kalimeri, K., Bonanomi, A., Beiro, M., Rosina, A., \& Cattuto, C. (2020). Traditional versus facebook-based surveys: Evaluation of biases in self-reported demographic and psychometric information.

Kühne, S., \& Zindel, Z. (2020). Using Facebook and Instagram to recruit web survey participants: A step-by-step guide and application. Survey Methods: Insights from the Field (SMIF).

Machado, N. M., Gomide, H. P., Bernardino, H. S., \& Ronzani, T. M. (2019). Facebook recruitment of smokers: Comparing gain-and loss-framed ads for the purposes of an internet-based smoking cessation intervention. Cadernos de saude publica, 35, e00151318.

Neundorf, A., \& Öztürk, A. (2021). Recruiting research participants through facebook: Assessing facebook advertisement tools. https://doi.org/10.31219/osf.io/3g74n

Oesterle, S., Epstein, M., Haggerty, K. P., \& Moreno, M. A. (2018). Using Facebook to recruit parents to participate in a family program to prevent teen drug use. Prevention Science, 19(4), 559-569.

Pham, K. H., Rampazzo, F., \& Rosenzweig, L. R. (2019). Online surveys and digital demography in the developing world: Facebook users in Kenya. arXiv preprint arXiv:1910.03448.

Pötzschke, S., \& Braun, M. (2017). Migrant sampling using Facebook advertisements: A case study of Polish migrants in four European countries. Social Science Computer Review, 35(5), 633-653.

Samuels, D. J., \& Zucco, C. (2013). Using Facebook as a subject recruitment tool for survey-experimental research. Available at SSRN 2101458.

Sances, M. W. (2018). Missing the target? using surveys to validate social media ad targeting. Political Science Research and Methods, 1-8. 
Schneider, D., \& Harknett, K. (2019). What's to like? Facebook as a tool for survey data collection. Sociological Methods \& Research, 0049124119882477.

Zhang, B., Mildenberger, M., Howe, P. D., Marlon, J., Rosenthal, S. A., \& Leiserowitz, A. (2020). Quota sampling using facebook advertisements. Political Science Research and Methods, 8(3), 558-564. 